\title{
WestVirginiaUniversity
}

THE RESEARCH REPOSITORY @ WVU

Graduate Theses, Dissertations, and Problem Reports

2004

\section{Correlates of 9-month-old infants' night awakening}

Cheryl Wiedman

West Virginia University

Follow this and additional works at: https://researchrepository.wvu.edu/etd

\section{Recommended Citation}

Wiedman, Cheryl, "Correlates of 9-month-old infants' night awakening" (2004). Graduate Theses,

Dissertations, and Problem Reports. 2094.

https://researchrepository.wvu.edu/etd/2094

This Dissertation is protected by copyright and/or related rights. It has been brought to you by the The Research Repository @ WVU with permission from the rights-holder(s). You are free to use this Dissertation in any way that is permitted by the copyright and related rights legislation that applies to your use. For other uses you must obtain permission from the rights-holder(s) directly, unless additional rights are indicated by a Creative Commons license in the record and/ or on the work itself. This Dissertation has been accepted for inclusion in WVU Graduate Theses, Dissertations, and Problem Reports collection by an authorized administrator of The Research Repository @ WVU.

For more information, please contact researchrepository@mail.wvu.edu. 


\title{
Correlates of 9-Month-Old Infants’ Night Awakening
}

\author{
Cheryl Wiedman
}

Dissertation submitted to the Eberly College of Arts and Sciences at West Virginia University in partial fulfillment of the requirements for the degree of

Doctor of Philosophy

\author{
Katherine Karraker, Ph.D., Chair \\ Lindsey Cohen, Ph.D. \\ Lesley Cottrell, Ph.D. \\ Deborah Jones, Ph.D. \\ Matthew Scullin, Ph.D \\ Life-Span Developmental Psychology
}

Morgantown, West Virginia

2004

Keywords: Infant night awakening, Maternal sleep deprivation 


\section{ABSTRACT \\ Correlates of 9-Month-Old Infants’ Night Awakening}

\section{Cheryl Wiedman}

There are individual differences in 9-month-old infants' night awakening behavior. Some infants are able to soothe themselves back to sleep however; others are unable to fall back asleep without parental intervention. Attending to a waking infant frequently during the night may cause problems for parents due to the effects of sleep deprivation. In particular, mothers of infants who are sleep deprived as a result of their infants' frequent night awakenings have been found to experience deficits in mood and perceptions of their infants. Given that there are individual differences in night awakening behavior the present study sought to determine: 1) whether infant characteristics were associated with night awakening and 2) whether variations in infant night awakening were related to infant behavior and maternal characteristics and behaviors. Specific factors were found to be related to infant night awakening in 9-month-olds. Care-giving factors such as feeding method, method used to put the infant to sleep, and cosleeping practices were all related to infant night awakening. Individual difference factors were also associated with infant night awakening. Infants who experienced separation anxiety, were dysregulated, napped more, and cried more experienced more night awakening than infants who did not have these characteristics. No individual difference factors in the mother were related to maternal perceptions of and expectations for the infant. However, variations in infant night awakening were related to individual differences in maternal characteristics. Mothers whose infants awakened more frequently during the night reported experiencing more sleep deprivation, more depressive symptoms, and lower levels of optimism. These findings can be used to guide future research, which could utilize experimental methods to ascertain whether infant night awakening can be reduced or eliminated by manipulating environmental conditions. If night awakening is reduced through these experimental methods then the difficulties associated with maternal sleep deprivation, such as deficits in mood and optimism, could also be alleviated. 


\section{Dedication}

This manuscript is dedicated in loving memory to my mother. I did it for both of us Mom. 


\section{Acknowledgements}

I would like to take this time to acknowledge all of the individuals who helped me complete this project.

First, I would like to thank my fiancé and family for providing me with the loving support and encouragement I needed during the duration of the project and throughout graduate school.

Second, I would like to thank Dr. Katherine Karraker for her mentorship and unending patience with my questions and concerns.

I would also like to thank my undergraduate adviser and lifetime mentor Dr. Robert Padgett. Thanks for always telling me “you can do it” Dr. P.

Finally, I would like to acknowledge the American Psychological Association and the Eberly College of Arts and Sciences for providing me with the funding necessary to complete this project. 


\section{Table of Contents}

Title Page

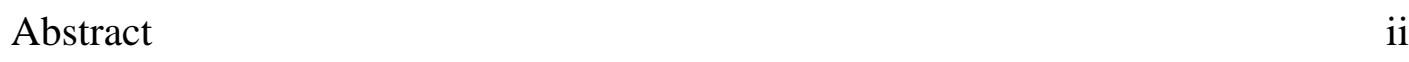

Dedication iii

Acknowledgements $\quad$ iv

Table of Contents $\quad$ v-vii

List of Tables $\quad$ viii-ix

List of Figures $\quad$ X

Introduction 1 1-26

Infant Regulation 3

Infant Night Awakening $\quad$ 4-8

Relations Between Infant Night Awakening and Infant Characteristics 8-13

Relations Between Infant Night Awakening and Maternal Characteristics 14-24

Statement of the Problem 24-25

Research Questions and Hypotheses $\quad$ 25-26

$\begin{array}{ll}\text { Method 27-34 } & \text { 27 }\end{array}$

$\begin{array}{ll}\text { Participants } & 27\end{array}$

$\begin{array}{lr}\text { Procedure } & \text { 27-29 }\end{array}$

$\begin{array}{lr}\text { Scoring of the BSID-II } & 29\end{array}$

$\begin{array}{lr}\text { Measures } & \text { 29-34 }\end{array}$

Results $34-48$

$\begin{array}{ll}\text { Initial Analyses } & \text { 34-38 }\end{array}$

$\begin{array}{ll}\text { Tests of Hypotheses } & \text { 38-44 }\end{array}$ 
Exploratory Analyses $\quad$ 45-48

Discussion 49-65

Infant Characteristics Associated with Night Awakening 49-52

Variations in Infant Night Awakening Related to Infant Behavior and Maternal

$\begin{array}{lr}\text { Characteristics and Behavior } & \text { 52-58 }\end{array}$

Other Factors Related to Infant Night Awakening 58-62

Maternal Perceptions of Infant Behavior 62-63

Limitations and Future Directions 63-65

$\begin{array}{ll}\text { Conclusion } & 65\end{array}$

References

$\begin{array}{ll}\text { Table } 1 & \text { 74-75 }\end{array}$

$\begin{array}{ll}\text { Table } 2 & 76\end{array}$

$\begin{array}{ll}\text { Table } 3 & 77\end{array}$

$\begin{array}{ll}\text { Table } 4 & \text { 78-79 }\end{array}$

$\begin{array}{ll}\text { Table } 5 & 80\end{array}$

$\begin{array}{lr}\text { Table } 6 & 81\end{array}$

$\begin{array}{ll}\text { Table } 7 & 82\end{array}$

Table $8 \quad 83$

$\begin{array}{ll}\text { Table } 9 & 84\end{array}$

$\begin{array}{ll}\text { Table } 10 & 85\end{array}$

Table $11 \quad 86$

$\begin{array}{ll}\text { Table } 12 & 87\end{array}$

$\begin{array}{ll}\text { Table } 13 & 88\end{array}$ 
Table 14

Table 15

92

Table 16

93

Table 17

94

Table 18

95

Figure 1

96-97

Appendix A: Background Questionnaire

98-103

Appendix B: Infant Care Diary Sample

104-106

Appendix C: Bayley Expectations Questionnaire

107-111

Appendix D: Expectation Paradigm

112-113 


\section{List of Tables}

Table $1 \quad$ Participant Characteristics 74-75

Table 2 Participant Demographic Variables 76

Table 3 Means, Standard Deviations, and Ranges for Variables in Figure 177

Table 4 Participant Means, Standard Deviations, and Ranges from the Infant Care Diary 78-79

Table 5 Night Awakening Differences During the Duration of Nighttime Sleep Between Infants Who Breast-Feed and Infants Who Bottle-Fed 80

Table 6 Night Awakening Differences During the Duration of Nighttime Sleep Between Infants Who Are Put To Bed Awake and Infants Who Are Put To Bed Asleep 81

Table $7 \quad$ Night Awakening Differences During the Duration of Nighttime Sleep Between Infants Who Co-Sleep and Infants Who Do Not Co-sleep 82

Table 8 Night Awakening Differences During the Duration of Nighttime Sleep Between Infants With Separation Anxiety and Infants Without Separation Anxiety 83

Table 9 Night Awakening Differences During the Duration of Nighttime Sleep Between Infants Who Use a Sleep Aid and Infant Who Do Not Use A Sleep Aid 84

Table 10 Night Awakening Differences During the Duration of Nighttime Sleep Between Infants Who Were Teething and Infants Who Were Not Teething 85

Table 11 Intercorrelations Between Infant Night Awakening During the Duration of Nighttime Sleep and Infant Temperament and Behavior 86

Table 12 Means, Standard Deviations, and Ranges for Maternal Expectation Variables 87 Table 13 Intercorrelations Between Maternal Perceptions of and Expectations for the Infant and Maternal Characteristics 88 
Table 14 Intercorrelations Between Infant Sleep Variables and Maternal Sleep Variables and Characteristics 89-91

Table 15 Intercorrelations Between Maternal and Principal Investigator (PI) Behavior Rating Scale Scores 92

Table 16 Means and Standard Deviations for Maternal Perceptions of Infant Manageability as a Function of Maternal Mood and Optimism 93

Table 17 Means and Standard Deviations for Maternal Expectations as a Function of Maternal Mood and Optimism 94

Table 18 Maternal Sleep Behavior Differences Between Mothers of First-Borns and Mothers of Later-Borns 95 


\section{List of Figures}

Figure 1 A model of the relations between infant night awakening and maternal and infant characteristics 96-97 


\section{Correlates of 9-Month-Old Infants’ Night Awakening}

In the first month of life almost all infants awaken during the night and require some parental intervention to return back to sleep (Anders, 1994). By 4 months of age, most infants continue to awaken during the night but are able to soothe themselves back to sleep without the aid of parents (Anders). However, some infants continue to be unable to soothe themselves back to sleep without parental intervention. Some waking infants are able to soothe themselves back to sleep in the early months but in the second half of the first year begin to require parental intervention again to soothe themselves back to sleep. This pattern of night awakening that requires parental intervention in the second half of the first year seems to peak at 9 months of age (Anders, 1979). These individual differences in infant night awakening behaviors suggest that there may be infant characteristics that are associated with night awakening. One goal of the present study was to determine if infant characteristics are associated with night awakening. Infants who differ in their night awakening also require different amounts of parental attention to help soothe them back to sleep. Attending to a waking infant frequently during the night may cause problems for parents due to the effects of sleep deprivation (Karraker \& Cottrell, 2000). In particular, mothers of young infants who are sleep deprived as a result of their infants’ frequent night awakenings have been found to experience deficits in mood and perceptions of their infants (Goodlin-Jones, Eiben, \& Anders, 1997; Nover, Shore, Timberlake, \& Greenspan, 1984). Given that there are individual differences in night awakening behavior another goal of the present study was to determine whether variations in infant night awakening were related to infant behavior and maternal characteristics and behaviors.

The focus of the present study was on 9-month-old infants and their mothers. Ninemonth-olds were targeted because previous research has suggested that there is a significant peak 
in night awakening at this age (Anders, 1979; Nover et al., 1984; Paret, 1983). Specifically, the present study examined the pattern of night awakening that requires parental attention due to infant signaling. This type of night awakening will be generally referred to as infant night awakening throughout the paper. The study was unique because it simultaneously examined infant and maternal variables that other studies (e.g., Morrell \& Steele, 2003) have suggested may be related to infant night awakening. Infant characteristics and behavior such as temperament, dysregulation, and cognitive performance were found to be related to night awakening behaviors in previous studies but had yet to be explored with a sample of healthy, normally developing 9-month-old infants. Infant night awakening was also found to be related to maternal sleep deprivation but few studies had examined how maternal sleep deprivation might be related to the mother's perceptions of her infant and no studies have examined how sleep deprivation might be related to the mother's expectations for her infant. Also, the possible mediating effects of maternal mood and the moderating effects of maternal optimism on the relations between maternal sleep deprivation and maternal perceptions of and expectations for the infant had yet to be explored.

It should be acknowledged that the correlational nature of this study did not permit certain variables from being identified as causes or effects of infant night awakening. However, the results of the study can be used to suggest certain causal relations that can be explored in subsequent studies examining the effects of night awakening on infant and maternal characteristics. Despite this limitation, research examining the correlates of night awakening in 9-month-old infants is important because it can be used to provide information to the public about infant behaviors that may be related to night awakening that could be targeted in subsequent intervention attempts. In order to better understand the mechanisms underlying infant 
night awakening, current knowledge about infant regulation, infant sleep patterns, and infant and maternal characteristics will be reviewed below.

\section{Infant Regulation}

Self-regulation in infancy is based on infants’ capacity to develop homeostasis during the first few months of their life while they are simultaneously regulating their arousal and coordinating their responses to sensory stimulation. As infants continue to develop, selfregulation becomes more dependent on their capacity to communicate using vocal and gestural signals as well as to internalize daily routines, and to be contingently responsive to the expectations of others (DeGangi, 2000). Prolonged periods of sleep states, smooth state transitions, low irritability, and integrated orientation to the appearance of visual or auditory stimuli are all indicators of regulation in infancy (DiPietro \& Porges, 1991). An infant is considered to be regulatory-disordered when the infant is behaviorally difficult and has disturbances in sleep, self-consoling, eating, attention and arousal, mood regulation, or transition between states (DeGangi, Poisson, Sickel, \& Wiener, 1995).

One indicator of poor regulation in infants and children that researchers studying dysregulation have focused on is sleep disturbances. Children who experience sleep disturbances frequently show high states of arousal, which prevent them from inhibiting their alert state to allow themselves to sleep (DeGangi, 2000). Because the child is unable to fall into a deep REM sleep they awaken frequently during the night. When this type of sleep disturbance is present, infants have difficulty regulating their sleep-wake cycles and subsequently have trouble falling and staying asleep (DeGangi). The present study examined infant night awakening as one aspect of infant dysregulation that was hypothesized to be related to infant characteristics and maternal sleep deprivation. 


\section{Infant Night Awakening}

Infant sleep patterns. At birth, healthy full-term newborns sleep for 16-18 of the 24 hours in a day and sleep-wake cycles tend to be between 3 and 4 hours in length. During the first month, sleep-wake cycles begin to adapt to light-dark cycles as well as other environmental cues. By the end of the first year, infants continue to sleep 14-15 hours per day. Infants are considered to be "sleeping through the night” if they sleep without removal from their crib between midnight and 5 AM for at least 4 weeks, whereas “night-waking” has been defined as arousal during that time with at least one weekly awakening for 4 weeks (Anders, 1979).

Awakening during the night has been found to be highly individualized. Bamford et al. (1990) reported that infants' total sleep and longest period of sleep were stable individual characteristics over time. They reported a high positive correlation between total sleep measured at 13, 26, and 52 weeks. They also reported that infant sleep patterns tended to be set early in their life and that wakeful infants tended to continue to be wakeful throughout their first year. Specifically, they found that the number of sleep episodes at 6 weeks was positively correlated with the number of sleep episodes at 13, 26, and 52 weeks. These results indicate that once infant sleep patterns have been established they appear to remain relatively stable throughout the first year. Therefore, some infants who have difficulties remaining asleep in the first weeks of their lives continue having difficulties in sleeping later, possibly adding to their parents’ distress and sleep deprivation.

The pattern of remaining asleep through the night begins around 2 months of age. By this time, infants' sleep patterns tend to be concentrated at night and waking normally occurs during the day. Problems arise when the process of nighttime consolidation fails to properly develop in some infants. These problems appear to be quite common given that by the time infants are 3 
months of age approximately half of them are routinely awakening their parents during the night (Karraker \& Cottrell, 2000). Between 3 and 6 months, the frequency of night awakening declines, in that most infants are able to "settle” themselves (Ferber, 1985). However, during the second half of the first year there is an increase in the number of night awakenings, with onethird to one-half of all infants this age waking during the night even if they did not have previous night waking problems (Anders, 1994; Anders, Keener, Bowe, \& Shoaff, 1983; Ferber; Mindell, 1997; Paret, 1983).

Nine months has been identified as the age when night awakening typically peaks in the second half of the first year (Anders, 1979; Nover et al. 1984; Paret, 1983). Moore and Ucko found in their longitudinal study of infant night awakening that of the 160 infants they sampled $10 \%$ of them were waking at 5 months. The prevalence of this night awakening rose to $20 \%$ at 9 months and then began to slowly decrease (as cited in Carey, 1974). At 9 months infants usually awaken after 6-7 hours of continuous sleep and approximately $60-80 \%$ of them are able to selfsoothe themselves back to sleep. However, it is those infants who are unable to self-soothe themselves back to sleep who become problematic for parents (Anders, 1994).

There are various theories as to why this increase in night awakening occurs during the second half of the first year. Paret (1983) suggested that night waking increased in 9-month-olds due to separation anxiety from the mother, acquisition of new motor skills, or the physical pain of teething.

Regardless of why some 9-month-old infants are no longer able to soothe themselves back to sleep, parents of these awakening infants likely experience the effects of partial sleep deprivation such as impairment of daytime functioning and mood (Dement, 1999). Researchers have found that mothers are more at risk for the deleterious effects of sleep deprivation than 
fathers because mothers awaken to care for their children more than fathers (Crowe, Clark, \& Qualls, 1996; Ferber, 1985).

Napping. By 4 months most infants take 3 naps per day and by 6 months the number of naps is reduced to 2 in that $90 \%$ of infants take one nap in the morning and one nap in the afternoon. By 9 months, most infants are still taking 2 naps per day; however some infants begin to take only 1 nap per day (Mindell, 1997). The evidence suggests that like night awakening, napping may be a highly individualized behavior. Therefore, the present study asked mothers to provide information about the number and length of naps that the 9-month-old infants were taking during the day to ascertain whether their napping behavior was related to their night awakening. Napping behavior was one of several variables thought to be correlated with infant night awakening. Other variables such as feeding method, method used to put the infant to sleep, co-sleeping, and genetics were also thought to be related to the frequency of infant night awakening (Burnham, Goodlin-Jones, Gaylor, \& Anders, 2002; Mindell, 1997).

Other correlates. Infants who are being breast-fed are much more likely to awaken during the night than infants who are bottle-fed. Burnham et al. (2002) conducted a longitudinal study that examined predictors of self-soothing behaviors from 1 to 12 months. The results of their study found that infants who were still being breast-fed at 12 months were less likely to self-soothe during the night than 12-month-old infants who had been weaned. These findings suggest that many infants associate nursing with falling asleep and therefore, when they awaken during the night they cannot fall back asleep without being fed (Mindell, 1997). These results indicate that breast-feeding is related to infant night awakening and therefore the present study asked mothers to indicate the method by which they feed their infants. 
Another correlate of infant night awakening is the method the parent uses to put the child to sleep. Research has found that putting the infant to bed awake is an important factor that contributes to whether or not the infant remains asleep during the night (Mindell, 1997). Burnham et al. (2002) found that infants who were placed in their cribs already asleep and who did not use a sleep aid spent more time awake during the night and were less able to self-soothe themselves back to sleep. This research suggests that these variables could be related to the infant's frequency of awakening. Therefore, mothers in the present study were asked to indicate whether their infant was put to bed awake or asleep and if the infant used a sleep aid (i.e. pacifier).

Past research has also found that co-sleeping is related to infant night awakening (McKenna et al., 1994; Mindell, 1997). Specifically, infants who co-sleep with their parents have been found to experience more difficulties in falling asleep without their parent's presence (Mindell). Infants who co-sleep with their parents also appear to be awakened more frequently during the night and spend less time in the deep stages of sleep (McKenna et al.). Given these findings, the current study also examined whether the infant co-slept with the mother and how much time per week the infant spent co-sleeping with the mother.

Finally, it was speculated that there might be a genetic component related to infant night awakening. Specifically, it was thought that parental sleep patterns could be related to infant sleep patterns due to shared genetic factors. Therefore, the present study not only assessed the mother's sleep patterns but also asked the mother to indicate the average amount of sleep her partner received as well as how often her partner awakened during the night. However, there are many environmental factors that could influence both infant and parental sleep. Therefore, it was 
difficult to ascertain whether parental sleep patterns were related to infant sleep patterns due to shared genetic or shared environmental factors.

The research presented above indicated that there is individual variation in night awakening across infancy and many factors can contribute to why an infant may awaken. How individual variations in infant night awakening are related to specific characteristics of the infant will be examined next.

Relations between Night Awakening and Infant Characteristics

Temperament. Sleeping patterns in children have been found to be related to several temperamental characteristics (Anders \& Keener, 1985; Carey, 1974; Owens-Stively et al. 1997; Scher, Epstein, Sadeh, Tirosh, \& Lavie, 1992; Scher, Tirosh, \& Lavie, 1998; Van Tassel, 1985; Zuckerman, Stevenson, \& Bailey, 1987). Carey was one of the first researchers who examined the relation between sleep and temperament. He found that there was a significant correlation between sleep disturbance and the temperamental characteristic of sensory threshold in a sample of 6-12-month-old infants. Carey speculated that infants with low sensory threshold experienced more sleep disturbances because the greater responses they experienced to stimuli during the day continued to make them more arousable at night.

Subsequent research exploring the relation between childhood sleep and parent- reported ratings of child temperament has both confirmed and refuted Carey's (1974) findings. In their study examining childhood sleep disorders and temperament Owens-Stively et al. (1997) found that the majority of studies they reviewed reported that sleep problems in infancy and childhood were related to lower levels of adaptability, rhythmicity, and sensory threshold, and to higher levels of intensity. Similarly, Van Tassel (1985) found that the temperament characteristics of adaptability and mood assessed during the child's first year of life significantly predicted 
sleeping disturbances during the child's second year. However, contrary to this evidence, Scher et al.’s $(1992,1998)$ research on sleep and temperament has suggested that the only dimension of infant temperament that consistently relates to infant sleep is rhythmicity.

Another explanation for the relation between infant temperament and night awakenings could be that fussy and irritable behaviors exhibited by the infant during the day are a result of the infant being overtired and unable to fall and stay asleep at night (DeGangi, 2000). That is, because they are fatigued they cry and fuss when awake (Weissbluth, 1989). Given this conflicting evidence, it appeared that more research needed to be conducted to investigate which temperamental characteristics were associated with sleep disturbances in infants.

Maternal ratings of infant temperament. One explanation for the conflicting findings in research examining the association between infant sleep and temperament is that the mother's biased perception of her infant may be masking or creating the relation between child temperament and sleep (Scher et al., 1998). Research examining maternal reports of infant temperament has revealed that parental perceptions consist of objective, subjective, and psychometric error components (Bates \& Bayles, 1984). This research suggests that maternal perceptions of infant temperament may not be indicative of the infant's actual behavior (Bates \& Bayles; Seifer, Sameroff, Barrett, \& Krafchuk, 1994). For example, Seifer et al. examined observer and maternal ratings of infant behavior once a week for 8 weeks. The relations between reliable observer ratings and maternal ratings of infant temperament were quite poor. Specifically, the largest correlation between maternal and observer ratings was only .48, $p<.05$. Based on these findings, the researchers concluded that parental reports of infant temperament were largely subjective and did not provide much information about the infant's actual behavior. 
One explanation for their findings is that parents are systematically biased about the behavior of their own children. In particular, factors such as socioeconomic status, race, anxiety, and depression have been found to create this bias (Seifer et al., 1994). Sameroff, Seifer, and Elias (1982) examined the relation between maternal characteristics, infant behavior, and maternal temperament ratings with a sample of 4-month-old infants. The results indicated that infants whose mothers were from lower-SES backgrounds, African American in origin, or mentally ill rated their infants as having more difficult temperaments than infants whose mothers did not have these characteristics. Similarly, Vaughn, Bradley, Joffe, Seifer, and Barglow (1987) found that mothers from low SES groups or who were African American in origin were more likely to rate their infants as more difficult than other mothers in the sample. Additionally, they found that maternal anxiety assessed prenatally was related to maternal ratings of infant temperament postnatally in that mothers who scored higher on the anxiety scale rated their infants as more difficult regardless of race.

Mothers in the present study completed ratings of their infant's temperament as well as behavior during a developmental assessment. The findings presented above suggested that there are limitations in using only maternal reports of infant temperament as indicators of infant actual behavior because they may not be accurate and may be influenced by maternal characteristics (Seifer et al, 1994; Vaughn et al., 1987). Therefore, in order to obtain more accurate information about the infant's actual behavior the present study used multiple measures of infant temperament and behavior in addition to the maternal reports.

One measure that was used to assess infant temperament was the infant's crying, which was recorded in an Infant Care Diary. Bates’s (1980) work using maternal ratings of infant temperament has revealed that the degree of an infant's difficultness can most easily be directly 
measured by assessing the frequency of infant crying and fussiness. Specifically, Lounsbury and Bates (1982) reported that $10 \%$ of the variance in maternal ratings of perceived difficulty could be accounted for by objectively recording the amount of crying the infant does. Therefore, the present study assessed the frequency of infant crying over one week to obtain a more objective measure of the infant's temperamental difficulty.

An additional measure of the infant's behavior, independent of maternal reports, was obtained by having the principal investigator complete ratings of the infant's behavior during a developmental assessment. This measure also allowed comparison of the principal investigator's ratings of the infant's actual behavior with the mother's perceptions of the infant's behavior, which may have been distorted.

Cognitive performance. Infant night awakening has also been found to relate to infant cognitive performance. A description of infant sleep states will be provided to help explain why this association may exist.

Infant sleep is characterized by three states. Infants are said to be in active sleep when their eyes are closed, their respiration is uneven, and they show frequent body movements and rapid eye movement, as well as smiles, sighs, mouthing, or sucking (Anders \& Keener, 1985; Thoman, 2001). Infants are said to be in quiet sleep when there is an absence of motor activity. Their eyes are closed, their respiration is slow and regular, and rhythmic mouthing is present (Anders \& Keener; Thoman). Active-quiet transition sleep is a state that occurs between active and quiet sleep in which infants show mixed behaviors that are characteristic of both active and quiet sleep. Their respiration fluctuates and they may let out sharp cries or vocalizations as they stretch (Thoman). Infant sleep state is important to consider because frequent awakening during certain sleep states has been found to have deleterious effects on infant development. 
Evidence suggests that infants whose sleep is fragmented (i.e. they do not spend enough time in certain sleep states) experience negative developmental outcomes. Research focusing on the three sleep states has found that premature infants who frequently awoke during the night, especially during quiet sleep, had lower mental development scores at 1 and 2 years of age than infants who did not experience these arousals during the night (Thoman, 2001). Premature infants are particularly prone to spending less time in quiet sleep. Beckwith and Parmelee (1986) examined the association between EEG patterns of premature infants at 40 weeks and their later cognitive development measured at 4, 9, and 24 months and again at 5 and 8 years. The results revealed that infants whose EEG patterns indicated less quiet sleep scored lower on developmental and IQ tests from 4 months to 8 years than those infants whose EEG patterns indicated that they spent more time in quiet sleep. The authors contended that the development of quiet sleep was an important indicator of brain development and organization because as the amount of quiet sleep increased so did the growth of dendrites, axons, and synapses in the brain (Beckwith \& Parmelee).

The association between sleep disruption and cognitive performance has also been demonstrated in a sample of preschool children. Bates, Viken, Alexander, Beyers, and Stockton (2002) examined the relation between sleep disruption and school adjustment in a large sample of lower income preschool students. The researchers utilized maternal reports of child sleep disruption for 4 weeks. The measures of disrupted sleep included sleep variability (within-child variance of hours of estimated sleep across the 4 weeks), bedtime variability (within-child variance in bedtime), and late bedtime (average nightly bedtime across the 4 weeks). Structural equation modeling was used to determine whether sleep and other variables (i.e. family management, family stress) influenced preschool adjustment. The results revealed that the 
child's disrupted sleep patterns (as measured by the three variables described above) were related to poor adjustment in preschool even after taking into consideration family stress and management practices. The authors concluded that because these sleep disrupted children were experiencing constant fatigue they were less adjusted in preschool due to cognitive disorientation and behavioral problems associated with being tired. These findings suggest that another way to measure sleep disruption is to examine the actual amount of time the child spends sleeping. According to these findings, it appears that sleep duration may also be related to the cognitive performance of children.

This collection of research findings, utilizing mostly premature infant and child samples, suggests that there are associations among quiet sleep, brain development, the amount of time spent sleeping, and subsequent cognitive performance. Infants who awaken frequently at night are spending less time in quiet sleep and their cognitive performance may be influenced. Therefore, the associations between infant night awakenings, the amount of time spent sleeping, and cognitive performance warranted some attention in the present study, especially in a sample of “normally” developing infants.

Summary. Infant night awakening is one aspect of infant dysregulation that is related to several infant characteristics and behaviors. Specifically, the collection of research findings presented above suggests that the frequency at which the infant awakens during the night and the total amount of time the infant spends sleeping are related to the infant's temperament and cognitive performance. Subsequent sections will highlight evidence that suggests several maternal characteristics that were hypothesized to be related to infant night awakening in the present study. 


\section{Relations between Infant Night Awakening and Maternal Characteristics}

Infants who differ in their night awakening behavior require different amounts of parental attention to help soothe them back to sleep. Given that mothers are more likely to attend to their waking infants during the night it was thought that there might be several maternal characteristics related to infant night awakening.

Maternal sleep deprivation. Research examining night awakening in infancy has found strong associations between infant night awakening and maternal reports of sleep deprivation. Wiedman (2002) sampled a group of 40 mothers and their 3-month-old infants and found significant differences between mothers classified as highly sleep deprived and those classified as not deprived. Mothers were classified as highly sleep deprived when the difference between the average amount of sleep they were receiving and the amount of sleep they reported they needed to function well was 2 hours or greater and the average number of times their infant awoke during the night was 2 or greater. Mothers were considered to be not sleep deprived when the difference between the average amount of sleep they were receiving and the amount of sleep they reported they needed to function well was 0 and the average number of times their infant awoke during the night was less than one. The results revealed that the groups significantly differed in their self-reported ratings of sleep deprivation, the number of times they were being awakened per week, how sleep deprivation was negatively affecting their functioning, and the number of naps they took per week. In particular, mothers in the highly sleep deprived group rated themselves as being more sleep deprived than the not deprived mothers and that their everyday functioning was being negatively affected by sleep deprivation more than the not deprived mothers (Wiedman). Clearly, this evidence suggests that mothers of infants who awaken frequently report that they are more sleep deprived than mothers of infants who do not 
awaken. In order to better understand how infant night awakening is related to the effects of sleep deprivation a discussion of how sleep deprivation affects maternal functioning is provided.

Research in the general adult population has revealed that sleep deprivation affects several aspects of an individual's behavior. Pilcher and Huffcutt (1996) performed a metaanalysis to summarize data from 19 experimental research studies that examined the effects of sleep deprivation on performance. Their analyses indicated that sleep deprivation could cause significant detriments in mood, cognition, and motor performance. The meta-analysis also revealed that partial sleep deprivation, resulting from fragmented sleep, was more deleterious than continuous sleep deprivation. Bonnett (1989) also found that fragmented sleep resulted in significant deficits in performance and mood. The more the individual's sleep was fragmented, the more deficits in performance and mood were exhibited. As the evidence indicated above, one population that is particularly subjected to fragmented sleep and the accompanying consequences are parents of young infants. Dement (1999) reported that parents of infants lose 2 hours of sleep per night over the first 5 months of infancy because infants sleep only about 8 fragmented hours per night. As a result parents may encounter many problems associated with sleep deprivation such as inability to focus, delayed and poor decision-making, indifference, and lack of motivation (Dement).

Maternal mood. Research also suggests that maternal sleep deprivation and fatigue are related to maternal mood, specifically symptoms of depression (Goodlin-Jones et al, 1997; Karraker \& Cottrell, 2000; Nover et al., 1984). Goodlin-Jones et al. examined maternal wellbeing and sleep-wake patterns in infants. They found that at 6 months, maternal well-being was significantly related to how mothers interacted with their infants at the start of the night, what occurred during the night, and whether the infant used a sleep aid. In particular, Goodlin-Jones et 
al. found that infants who were less likely to self-soothe during the night had mothers who reported higher levels of depressive feelings and stress. Another more recent study examining the impact of infant epilepsy on maternal functioning found that mothers' nighttime awakening was positively related to maternal depression and negatively related to mothers' perceptions of their sleep quality, marital satisfaction, and health (Cottrell, Atkins, \& Khan, 2002). Given these findings, sleep deprived mothers are evidently exhibiting depressive symptoms more than mothers who are not sleep deprived.

Perceptions of the infant. The collection of findings presented above indicated that there was an association between sleep deprivation and mood. Research also suggests that there is a relation between mood and negative perceptions of the infant. Specifically, several studies have found that mothers who are experiencing deficits in mood and sleep rate their infants more negatively than mothers who are not experiencing these deficits (Field, 1995; Foreman \& Henshaw, 2002; Karraker \& Cottrell, 2000; Nover et al., 1984; Wiedman, 2002).

One population that experiences deficits in mood and has been extensively studied is women diagnosed with postpartum depression (PPD). Women who experience PPD have consistently exhibited negative perceptions of their infants. Field (1995) reported that when PPD mothers and observers coded infant behaviors during an interaction the depressed mothers coded their infant's behaviors as more negative than did the observers. Similarly, Foreman and Henshaw (2002) found that they could distinguish between PPD mothers and non-PPD mothers by asking them 20 questions about their perceptions of their 6-month-old infants. Specifically, PPD mothers had a less positive view about their infants than non-PPD mothers.

The evidence also suggests that maternal perceptions of the infant influence motherinfant interaction and maternal affect even in mothers who have not been clinically diagnosed 
with a mood disorder. Halpern and McLean (1997) found that women who characterized their infants as fussier, unadaptable, and unpredictable reported feeling more psychologically distressed and were less involved during a play interaction with their infants. In another study, Nover et al. (1984) examined the relation between maternal perceptions and mother-infant interaction by obtaining a measure of maternal distortion of her infant by calculating the difference between the mother's rating of her infant's behavior during a play session and an observer’s rating of the infant's behavior during the play session. Larger difference scores indicated more distortion. The results revealed that mothers whose perceptions of their infants were distorted were less interactive, sociable, physically affectionate, and contingently responsive toward their infants during the play interaction than mothers whose perceptions were not distorted.

Negative maternal perceptions of the infant have also been found to be related to maternal sleep deprivation. Nover et al. (1984) found that mothers' negative perceptions of their infants' behavior during a play interaction were also associated with night awakening in their 9month-old infants. Evidence of the relation between maternal perceptions and sleep deprivation has also been found with 3-month-olds. Highly sleep deprived mothers rated their infants as less manageable than not sleep deprived mothers (Karraker \& Cottrell, 2000, Wiedman, 2002). This collection of findings indicates that maternal sleep deprivation resulting from infant night awakening is related to the mother's mood and perception of her infant.

Expectations of the infant. Regardless of the amount of sleep they are receiving, all mothers have expectations about their infant's behavior. A substantial amount of research has examined the relation between parental expectations for their child's abilities and the child's actual development (Stoiber, 1992). This research is based on the perspective that how parents' 
think about their child's abilities influences their behavior toward the child, which subsequently influences the child's development (Stoiber). Typically, parental expectations are measured in terms of overestimations, underestimations, and accuracies. Most parents are above chance in their evaluation of their child's abilities (Miller, Manhal, \& Mee, 1991). Usually when parents make mistakes in predicting their child's abilities it is because they overestimate their child's performance (Miller, 1988; Stoiber). For example, Stoiber examined a sample of preschool children and their mothers' expectations for the child's performance on cognitive, motor, and social tasks. The results revealed that mothers were less accurate at predicting their child's performance on the social tasks in comparison to the cognitive or motor tasks. Mothers overestimated their child's social abilities more than their cognitive or motor abilities.

Miller (1988) reviewed the literature examining the association between parents' beliefs and children's cognitive development and concluded that, based on the evidence, parental overestimations, underestimations, and accuracies about their child's abilities are related to the parent's behavior toward the child. Miller's review revealed that more adaptive child rearing beliefs were associated with more accurate conceptions about the child's abilities. He also found that parents' beliefs about their child's cognitive development predicted the child's actual performance. For example, Seifer and Sameroff (as cited in Miller) found that maternal beliefs significantly predicted the child's IQ even after other variables were controlled. Another study found that the more inaccurate the mother's judgments of her child's abilities were, the less well the child performed on IQ items (Hunt \& Paraskevopoulos, 1980). This finding has been replicated with other samples as well. For example, mothers of boys who had a learning disability had lower expectations for their sons’ performance and more negative nonverbal behavior toward them during an academic task that they were asked to teach their sons. 
Subsequent analyses revealed that mothers who had negative expectations of their son's performance had sons who actually scored lower on the learning task (Tollison, Palmer, \& Stowe, 1987). This finding has even been replicated with normally developing infants. Karraker and Coleman (1999) found that mothers who expected their infants to pass many of the items on the Bayley Scales of Infant Development had toddlers who scored higher on the Mental Development Index.

Parental overestimations, underestimations, and accuracies have also been found to be related to children's behavior in other domains as well. For example, research has found that adolescent mothers who have more accurate expectations about their parenting, their children, and the parent-child relationship had children who displayed better coping skills than children of adolescent mothers who had unrealistic expectations (Stoiber \& Houghton, 1993). The contention among researchers in this area is that the more accurate the parent, the better the child's developmental level (Miller et al., 1991; Stoiber, 1992). One explanation that researchers give for the relation between parental beliefs and child outcomes is that parents who are knowledgeable about developmental sequences provide their child with an environment that facilitates their child’s developing social and cognitive abilities (Tamis-LaMonda, Chen, \& Bornstein, 1998). This “match hypothesis” asserts that the greater the parent's knowledge about the child the better the match between their teaching level and the child's current and subsequent development (Hunt \& Paraskevopoulos, 1980; Miller, 1988; Miller et al.). Based on her knowledge of the child, if the mother expects too little the child may not be stimulated enough or if she expects too much she may be overstimulating (Karraker \& Evans, 1995).

Summary. The evidence suggests that there is a relation between maternal expectations and child outcomes. The relation between sleep deprivation and maternal expectations has not 
been explored. However, based on the evidence provided above, it follows that if the mother's perceptions of her infant are more negative when she is sleep deprived then her expectations of her infant may also be distorted, which might affect the mother's behavior and then the child's subsequent performance and possibly his or her development. One additional factor that was hypothesized to influence a mother's perception of and expectations for her infant in the present study was her personality.

Maternal personality and perceptions of and expectations for the infant. The evidence provided in the previous section illustrated that maternal sleep deprivation is likely to be related to maternal perceptions of, and possibly expectations for, the infant. However, other maternal characteristics also may be related to her perceptions of and expectations for her infant besides sleep deprivation. One such characteristic is personality. Specifically, certain characteristics of the mother’s personality were thought to influence the relation between maternal sleep deprivation and maternal perceptions of and expectations for her infant in the present study.

The association between personality and parenting has been extensively studied. Belsky’s (1984) seminal work described three domains of determinants that influenced how the parent interacts with the child that included: personal psychological resources of the parent, characteristics of the child, and contextual sources of stress and support. However, for the purpose of the present discussion only individual parent characteristics will be discussed. The model assumed that parenting was directly influenced by characteristics within the parent (i.e. personality). Subsequent research utilizing Belsky’s model has found that mothers who were high in certain personality characteristics, such as neuroticism, showed less positive affect, were less sensitive and cognitively stimulating, and were more intrusive toward their infants than other women (Belsky, Crnic, \& Woodworth, 1995). Other research has demonstrated that mothers who 
were high in neuroticism also used more controlling discipline with their children than other mothers (Clark, Kochanska, \& Ready, 2000). These findings indicate that characteristics of a mother's personality can be used to predict some parenting behaviors (Kochanska, Clark, \& Goldman, 1997).

One personality characteristic, optimism, has been overlooked in the literature in terms of its relation to parenting (Jones, Forehand, Brody, \& Armistead 2002; Peterson, 2000). In general, optimists typically report having lives free from pressures and annoyances, getting greater satisfaction from friendships and family relations, as well as having greater job satisfaction. Individuals who are high in optimism engage in more health-enhancing behaviors and utilize better coping strategies in that they are more problem-focused. Optimists confront problems in a realistic manner and take active steps to make their situations better and do not try to wish their problems away (Scheier \& Carver, 1985, 1992). Scheier and Carver reported that individuals who were optimistic tended to continue to be optimistic across time and contexts. This statement lends itself to the argument that optimism is a trait rather than a state that may vary depending on the circumstances. In other words, optimists tend to be optimistic in general and have positive expectations regardless of the context.

Given that optimists are more positive and tend to believe that good, rather than bad, things will happen to them in all domains, it is likely that optimism influences parenting domains as well. Jones et al. (2002) examined whether maternal optimism was related to positive parenting and child outcomes in a sample of inner-city, single-parent African American families. They hypothesized that optimism would be a protective factor for single mothers living in difficult conditions. Specifically, the authors hypothesized that mothers who were more optimistic would show more positive parenting behaviors. Their findings indicated that maternal 
optimism significantly predicted positive parenting in that mothers who were high in optimism monitored their child's behaviors more and had better mother-child relations than mothers who were low in optimism.

In summary, these findings can be applied to the present study, which explored the relation between maternal optimism and sleep deprived mother's perceptions of and expectations for their infants. The association between aspects of maternal personality, such as neuroticism and extraversion, and parenting has already been explored in the literature. However, other aspects of maternal personality such as optimism have minimally been explored and it was thought that given the characteristics optimists typically possess (i.e. they are more positive across time and situations) there would be relations between this aspect of the mother's personality, sleep deprivation, and her perceptions of and expectations for her infant. It was thought that sleep deprived mothers who were high in optimism would be more accepting of the sleep deprivation they were experiencing as a result of their infant's night awakening than sleep deprived mothers who were low in optimism. Based on the research that found a significant relation between maternal optimism and positive parenting, it was also thought that mothers high in optimism would be more positive toward their infants than mothers low in optimism.

Mediating and Moderating Variables. The evidence presented above suggests that there are relations between maternal sleep deprivation and maternal perceptions of and expectations for the infant that may be influenced by certain maternal characteristics such as her mood and degree of optimism. Therefore, the present study examined how the mediating effects of maternal mood and the moderating effects of degree of optimism were influencing the relations between maternal sleep deprivation and maternal perceptions of and expectations for the infant. 
The mediating effects of maternal mood were examined because the collection of research findings presented above suggested that maternal mood was related to both sleep deprivation and perceptions of the infant. Therefore, it was thought that the effects of sleep deprivation on the mother's perceptions of and expectations for her infant could be accounted for by taking into consideration the mother's mood. A variable is considered to be a mediator when it meets the following conditions. First, variation in levels of the independent variable must be significantly accounted for by variation in the mediator (Baron \& Kenny, 1986). The evidence provided above suggested that variation in maternal sleep deprivation should be related to variation in maternal mood. Second, variation in levels of the mediator must be significantly accounted for by variation in the dependent variable (Baron \& Kenny). Variation in maternal mood has been found to be related to variation in perceptions of the infant. The final condition states that when the first two conditions are controlled for, the relation between the independent and dependent variables should no longer be significant (Baron \& Kenny). The present study explored whether the relations between maternal sleep deprivation and perceptions of and expectations for the infant would be reduced when the relations between maternal sleep deprivation and maternal mood as well as maternal mood and maternal perceptions were controlled.

Maternal optimism was measured and considered as a moderator of the relation between maternal sleep deprivation and maternal perception of and expectations for the infant because optimism has been shown to be related to a more positive outlook on life and parenting behaviors. Therefore, it was hypothesized that the mother's degree of optimism would influence the relation between her degree of sleep deprivation and her perceptions of and expectations for her infant. According to Baron and Kenny (1986), a moderator variable is one that "affects the 
direction and/or strength of the relation between the independent variable and the dependent variable” (p. 1174). Based on the optimism research described above it was thought that the mother's level of optimism would affect the strength of the relation between her level of sleep deprivation and the perceptions of and expectations for her infant because individuals who are optimistic have more positive perceptions regardless of the domain. Specifically, it was expected that the relation between maternal sleep deprivation and perceptions of and expectations for the infant would be weaker when the mother was high in optimism and the relation between maternal sleep deprivation and perceptions of and expectations for the infant would be stronger when the mother was low in optimism.

\section{Statement of the Problem}

The evidence presented above suggests that infant night awakening is related to difficulties in infant temperament and cognitive performance. The evidence also suggests that infant night awakening is related to maternal sleep deprivation, which may be related to her mood as well as perceptions of and expectations for her infant. Therefore, the main goal of the present study was to examine the relations between the variables indicated in the figure below (see Figure 1). Given the difficulty of recruiting large numbers of mother-infant dyads the causal ordering among the variables outlined in the figure was not tested. However, significant relations found between some of the variables indicated in the figure can be used to provide support for possible causal relations that could be explored in subsequent studies.

The study was limited because the mechanisms that were causing infant night awakening could not be directly tested. For example, it was possible that some infants awoke because they had difficult temperaments. It was also possible that some infants were difficult because they awoke frequently during the night. Due to the nature of this research it was difficult to assess 
causality because random assignment of infants to temperaments, regulatory styles, and sleep patterns was not possible.

Despite these limitations there is an important need for research in this area in order to provide education about infant night awakening to the public. The variables presented in the figure have been independently examined by different studies but have never been examined together with a sample of healthy, normally developing 9-month-old infants. Specifically, certain relations such as the mediating effects of maternal mood and the moderating effects of maternal optimism on relations between maternal sleep deprivation and maternal perceptions of and expectations for the infant had not been examined. The findings of the present study could be used to inform experimental research, which could target specific infant behaviors observed in the environment and could prevent infant night awakening from occurring. Perhaps if infant night awakening is decreased then the difficulties associated with maternal sleep deprivation could also be alleviated.

\section{Research Questions and Hypotheses}

The present study sought to explore how infant night awakening was related to both infant and maternal characteristics. To do so it attempted to answer the following questions and provide support for the corresponding hypotheses. 1) Is there a relation between night awakening and infant temperament? It was hypothesized that infants who awoke more during the night would be rated as more difficult by their mothers, cry more, and be “outside the normal limits” on the Behavior Rating Scale more than infants who awoke less during the night. 2) Is infant night awakening related to cognitive performance? It was predicted that infants who spent more time awake and frequently awoke during the night would score lower on the Mental Development Index of the Bayley Scales of Infant Development-II than infants who awoke less 
during the night. 3) Is maternal sleep deprivation related to perceptions of the infant? It was predicted that mothers who were more sleep deprived would rate their infants as more difficult on the temperament questionnaire, independent of their infant's crying behavior indicated on the Infant Care Diary and ratings of the infant's behavior during the administration of the Bayley Scales of Infant Development-II, than mothers who were less sleep deprived. 4) Is maternal sleep deprivation related to expectations for her infant? It was predicted that mothers who were more sleep deprived would underestimate their infant's performance on the mental development items of the Bayley Scales of Infant Development-II more than mothers who were less sleep deprived. 5) Is maternal optimism related to maternal perceptions of and expectations for her infant? It was predicted that maternal optimism would be related to more positive perceptions of and higher expectations for her infant. 6) Are the effects of sleep deprivation on maternal perceptions of and expectations for her infant moderated by maternal optimism? It was predicted that the mothers' level of optimism would affect the strength of the relation between her level of sleep deprivation and the perceptions and expectations she had for her infant. Specifically, it was hypothesized that the relation between maternal sleep deprivation and perceptions of and expectations for the infant would be weaker when the mother was high in optimism and the relation between maternal sleep deprivation and perceptions of and expectation for the infant would be stronger when the mother was low in optimism. 7) Are the effects of sleep deprivation on maternal perceptions of and expectations for her infant partially mediated by maternal mood? It was predicted that when the relations between maternal sleep deprivation and mood as well as mood and perceptions of and expectations for her infant were controlled, the relation between maternal sleep deprivation and perceptions of and expectations for her infant would be reduced. 


\section{Method}

\section{Participants}

A power analysis, using Sample Power, indicated that with a sample size of 40 and an alpha set at .05 the study would have power of .80. A total of 100 mothers were sent letters and/or contacted by a telephone call. Sixty-one of the mothers were reached and forty-five agreed to participate. However, four mothers withdrew from the study due to time constraints. Forty-one mothers and their 9-month-old infants (26 males and 15 females) living in the Morgantown area participated in the study. Nine-month-old infants were recruited because previous research suggested that there is a significant peak in night awakening at this age (Anders, 1979; Nover et al., 1984; Paret, 1983). Information gathered from the Infant Care Diary revealed that there was a substantial amount of infant night awakening occurring in this sample given that $42 \%$ of the infants were waking an average of one or more times per night.

To control for problems associated with special cases such as prematurity (less than 37 gestational weeks) and other medical problems (i.e. any malady that required hospitalization for at least one week), only women who delivered healthy full-term infants without complications were included in the study. County birth records were accessed to obtain the names of community members who had age appropriate infants. Addresses and phone numbers were obtained from the local telephone book.

\section{Procedure}

Mothers were initially contacted using an introductory letter describing the study when the infant was 8 months old. The letter was followed by a telephone call when the infant was $81 / 2$ months of age to inquire about the mother's interest in participation and to schedule a home visit. Mothers who agreed to participate were sent an information packet containing a letter describing 
the study in more detail, consent forms, appointment time, and several questionnaires to be completed the week prior to the home visit. Mothers were asked to complete an Infant Care Diary, as well as questionnaires that assessed her perceptions of her infant's temperament and dysregulation as well as her degree of optimism.

The home visit occurred approximately 2 weeks after the initial phone call. Visits were scheduled during the afternoon or early evening hours to control for any variance between participants that might have resulted from testing at different times of the day. Upon arrival at the home the primary investigator gave the mother a questionnaire that assessed her expectations for her infant's performance on the mental and motor development items of the Bayley Scales of Infant Development-II (BSID-II). The questionnaire asked her to indicate which items she thought her infant would successfully complete.

The principal investigator busied herself setting up a video camera and the BSID-II materials while the mother completed the expectation questionnaire so that she would be blind to the mothers' expectations of their infants' performance during the BSID-II administration. Upon completion of the questionnaire, the infant's current cognitive and motor development was assessed via the BSID-II. The recruitment procedure described above insured that all infants would be tested when their age was between 8 months and 16 days and 9 months and 15 days so that all infants in the sample received the same item set. The administration of the BSID-II took approximately 30 minutes and was videotaped for later scoring of the items if needed.

After the assessment was completed the mother was given several questionnaires to complete, which assessed her infant's behavior during the BSID-II, background information, and mood. Upon completion of the questionnaires, which took approximately 30 minutes, the dyad 
was compensated for their time. The infant was given a t-shirt and certificate of participation and the mother was given $\$ 20$.

Scoring of the BSID-II

Mental development index and psychomotor development index. The infant's performance on the mental development and psychomotor development items was scored during the administration of the items. Each item was scored as credit or no credit based on the guidelines for administration outlined in the BSID-II manual. The infant's raw score on the mental and psychomotor development items was calculated and converted into mental development index (MDI) and psychomotor development index (PDI) scores utilizing the appropriate appendixes located in the BSID-II manual. The scores were used as indexes of the infant's current developmental status (Bayley, 1993). For the purpose of the present study, only the infants’ MDI scores were utilized.

Behavior rating scale. Both the mother and primary investigator completed the Behavior Rating Scale (BRS), which assessed the infant's behavior during testing. The BRS asked questions pertaining to the administrator and mother's opinion of the infant's orientation and engagement, emotional regulation, and motor quality during the assessment. Raw scores were calculated, according to the manual instructions and indicated the extent to which the infant's behavior during the administration was within normal limits for his or her age, questionable, or non-optimal (Bayley, 1993).

\section{Measures}

In order to obtain information about the mother, her infant, and her household, mothers completed a background questionnaire on the day of the home visit. Specifically, she was asked questions about the average amount of sleep she received per night and the amount of sleep she 
needed to function well. She was also asked to indicate the type of feeding method she used to feed her infant, how many times her infant awakened per night, whether she put her infant to bed at night awake or asleep and if she co-slept with her infant. She also was asked questions about her partner’s sleeping patterns. Finally, she was asked to provide information about race, education, income, and employment status (see Appendix A).

The Infant Care Diary (ICD) (Karraker \& Cottrell, 2000) was used as another measure to assess maternal and infant sleep patterns one week prior to the home visit. Mothers who utilized day care or babysitters to provide care for their infants were asked to have the child care worker complete the ICD during the time that the infant was away from the mother. Instructions for completing the diary and a sample page from it are located in Appendix B.

From this measure the mother's total sleep time per night, total wake time per night, total wake frequency per night, and total nap time and frequency per day were calculated. The mothers’ average amount of sleep per night was calculated from this diary and subtracted from the number of hours she reported that she needed to function well on the background questionnaire. The difference between these numbers was used as an indicator of sleep deprivation (Karraker \& Cottrell, 2000). Measures of the infant's total sleep and wake times, total wake frequencies per night, and total time and frequency spent napping per day were also calculated. In addition, infant total crying time and crying frequency during the day and night were calculated in order to use them as measures of infant temperamental difficulty.

Infant night awakening was measured by calculating both the frequency of night awakenings and the amount of time the infant spent awake at night. Two types of infant night awakening were calculated using information from the ICD, infant night awakening during the duration of nighttime sleep and infant night awakening during maternal sleep. Infant night 
awakening during the duration of nighttime sleep was defined as the number of times and the amount of time the infant awoke during the period in which they were put to bed in the evening and awoke for the day the following morning. Infant night awakening during maternal sleep was defined as the number of times and the amount of time the infant awoke while their mother was sleeping during the night. Infant night awakening during maternal sleep and infant crying during the night were counted only when the mother was sleeping.

Maternal expectations for infant performance on the BSID-II were assessed during the home visit utilizing a Bayley Expectations Questionnaire. The questionnaire listed the age appropriate items from the MDI and PDI and asked the mother to indicate which items she thought her infant would successfully pass (see Appendix C). For the purpose of the present study, only the mother's expectations for her infant's performance on the mental items were utilized.

To assess maternal perceptions of her infant the Revised Infant Temperament Questionnaire (RITQ) (Carey Temperament Scales, 1996) was sent to the mother to be completed before the home visit. The mother was asked to complete the 95-item questionnaire by rating her infant on 6-point Likert scales yielding ratings for activity, rhythmicity, approach, adaptability, intensity, mood, persistence, distractibility, and threshold. The authors report the internal consistency, using coefficient alpha, of these scales to be .64, .65, .71, .57, .56, .53, .62, .49 , and .57 respectively. At the end of the questionnaire mothers were asked to rate several general impressions of their infants' temperament. The general impression scales included items pertaining to the infant’s activity, rhythmicity, approach, adaptability, intensity, mood, distractibility, attention span, and threshold as well as how manageable they thought their infant is in comparison with other infants of the same age. 
Mothers were also asked to complete the Infant Toddler Symptoms Checklist (DeGangi, 2000) during the week prior to the home visit as another measure of how regulated they perceived their infant to be. The checklist asked questions that pertain to the infant's sleep, selfcalming, feeding, sensory responses, communication and language, as well as emotional responses. Each question on the checklist was answered as “yes, most times," "past," or “never or sometimes.” Points were assigned to each response and totaled to determine if the infant was at risk for a regulatory disorder. The checklist has been validated on samples of normal and regulatory-disordered infants ranging from 7 to 30 months (DeGangi et al., 1995). The predictive validity of the checklist has been reported to be good, in that $78 \%$ of infants who were identified as having problems via the checklist were later diagnosed with having developmental or behavioral problems at 3 years (DeGangi, 2000). The internal consistency, using coefficient alpha, for the present study was .80 .

To ascertain the extent to which sleep deprived mothers were experiencing depressive symptoms, the Beck Depression Inventory-II (BDI-II) was administered following the BSID-II administration. This measure is a frequently used depression assessment that requires participants to rate their feelings on a 4-point Likert scale ranging from 0 to 3. Individuals with scores of 20 or above are considered to be suffering from moderate levels of depression. Individuals with scores of 9 or lower are considered to be nondepressed. According to this criterion, $71 \%$ of mothers in the present sample were nondepressed. Beck, Steer, and Garbin (1988) reported the internal consistency, using coefficient alpha, for the BDI-II to be .92 for outpatient populations and .93 for college students. The internal consistency, using coefficient alpha, for the present study was .91. 
The Life Orientation Test-Revised (LOT-R) was used to assess maternal optimism (Scheier, Carver, \& Bridges, 1994). The LOT-R is composed of 10 statements, 6 of which are used to derive an optimism score and the remaining 4 of which are fillers. Individuals were asked to rate the extent of their agreement with each statement on a 4-point scale ranging from 0 "strongly disagree" to 4 "strongly agree." Responses to the 6 optimism questions were then totaled to yield an overall optimism score, with higher scores indicating more optimism. The authors reported the mean score for a sample of 2,055 college students to be 14.33 for women and 14.42 for men. Scheier et al. reported the internal consistency, using coefficient alpha, of the entire 6 items to be .78 and the test-retest reliability at 4, 12, 24, and 28 months to be $.68, .60$, .56, and .79 respectively. In the present study the internal consistency of the entire 6 items, using coefficient alpha, was .86.

In order to assess the infant's cognitive performance the BSID-II was used. The BSID-II is used to assess the current development of infants and toddlers from 1 to 42 months. The scales are individually administered in the presence of one of the infant's parents. The infant is assessed using items that are part of an age-appropriate item set. The age-appropriate items administered to infants in the present study are listed in Appendix C. Items from the mental scale assess the infant's cognitive, language, and social skills. Items from the motor scale assess the infant's gross and fine motor skills. After the mental and motor scales are administered the individual performing the assessment completes a behavior rating scale, which assesses the infant's attention, engagement, emotional regulation, motor quality, and social responsiveness during testing (Bayley, 1993).

The BSID-II was standardized on a sample of 1,700 children with 100 children in 17 groups ranging from 1 to 42 months. The authors reported that the reliabilities, using coefficient 
alpha, for the 9-month-old item sets for the MDI and PDI are .84 and .85 respectively. The reliabilities, using coefficient alpha, for the orientation and engagement, emotional regulation, and motor quality subscales of the BRS are .87, .75, and .80 respectively (Bayley, 1993).

\section{Results}

\section{Initial Analyses}

Descriptive statistics. Descriptive statistics providing information about the sample are reported in Tables 1 and 2. Mothers who participated in the study were mainly non-Hispanic White and all were married to the father of the infant. Over half of the mothers were employed and the others were homemakers. The mothers were highly educated given that the majority of the sample had some college experience or higher. Infants in the sample were mainly later-borns and most did not attend day care.

Means, standard deviations, and ranges for the variables displayed in Figure 1 are provided in Table 3. An examination of the means revealed that they were in the expected ranges according to the authors of the scales. DeGangi (2000) reported that infants who score 10 or higher on the Infant Toddler Symptom Checklist are considered to be at risk for a regulatory disorder. An examination of the mean for the present sample revealed that the infants were generally not dysregulated. Bayley (1993) reported that infants were within the normal limits when their developmental index scores fell between 85 and 114. The mean for the sample indicated that the infants' mental development index scores were within the normal limits. According to Beck et al. (1988) individuals with scores of 9 or lower on the BDI are considered to be nondepressed. The mean BDI score for the sample was below that cutoff score. The mean for maternal optimism was above Scheier et al's (1994) mean for optimism. As a sample the mothers did not appear to be experiencing depressive symptoms and were high in optimism. 
Daily averages were computed using data acquired from the ICD to obtain information about infant behavior such as total time spent sleeping per night, total time spent awake during the duration of nighttime sleep per night, total time spent awake during maternal sleep per night, total number of awakenings during the duration of nighttime sleep per night, total number of awakening during maternal sleep per night, total time spent crying per day and night, total number of crying episodes per day and night, total time spent napping, and total number of naps taken per day.

Daily averages were also computed to obtain information about maternal behavior such as total sleep per night, total time spent awake per night, total number of times awakened per night, total amount of time spent napping, and total number of naps taken per day (see Table 4). A continuous maternal sleep deprivation variable was also calculated by subtracting the mother's average amount of sleep per night derived from the ICD from the number of hours of sleep she reported that she needs to function well on the background questionnaire (see Table 3).

A review of the means calculated from the ICD revealed that infants in the present sample were receiving approximately 10 hours of sleep and were waking 1 time per night on average, which is consistent with past research with 9-month-old infant samples (Anders, 1979). The mothers in the sample were receiving approximately 7 hours of sleep per night, which is less than the recommended amount of sleep for adults (Santrock, 2002).

Factors related to infant night awakening. Past research described above suggested that certain infant and parent variables are related to infant night awakening. Therefore, t-tests were conducted to determine whether there were significant differences in infant night awakening during the duration of nighttime sleep between infants who experience differences in feeding methods, methods their parents used to put them to sleep, use of a sleep aid, co-sleeping, 
separation anxiety, and teething. Correlations were conducted to assess the association between infant napping behavior and night awakening during the duration of nighttime sleep as measured by the ICD. Correlations were also conducted to determine whether there were relations between fathers' average amount of sleep per night and average awakenings per night and infant night awakening during the duration of nighttime sleep. An alpha level of .05 was used with all of the statistical analyses.

Feeding method. Thirty-seven percent of the infants in the sample were breast-fed and there were significant differences in infant night awakening during the duration of nighttime sleep between infants who were breast-fed and infants who were bottle-fed. Infants who were breast-fed awakened more frequently during the duration of nighttime sleep than infants who were bottle-fed (see Table 5).

Method put to sleep. Forty-four percent of the mothers reported that they put their infants to bed asleep at night. There were significant differences in night awakening during the duration of nighttime sleep between infants who were put to bed asleep and infants who were put to bed awake. Infants who were put to bed asleep spent more time awake during the duration of nighttime sleep in comparison to infants who were put to bed awake (see Table 6).

Co-sleeping. Co-sleeping was assessed by asking the mother whether the infant slept in bed with her. Twenty-four percent of mothers in the sample indicated that they co-slept with their infants ranging from 1-7 times per week $(M=5.30, S D=2.58)$. There were significant differences between infants who co-slept with their mothers and those who did not co-sleep with their mothers. Infants who co-slept with their mothers awakened significantly more frequently during the duration of nighttime sleep and spent more time awake at night during the duration of nighttime sleep than infants who did not co-sleep with their mothers (see Table 7). 
Separation anxiety. Significant differences were also found between infants whose mothers indicated that the infants were experiencing separation anxiety (61\%) and infants whose mothers indicated that the infants were not experiencing separation anxiety. Infants who were experiencing separation anxiety awakened more frequently and spent more time awake during the duration of nighttime sleep than infants who were not experiencing separation anxiety (see Table 8).

Use of sleep aid. There were no significant differences in infant night awakening during the duration of nighttime sleep between infants who were using a sleep aid at night (66\%) and infants who were not using a sleep aid (see Table 9). However, the difference in the number of times the infant awoke during the night between infants who used a sleep aid and infants who did not use a sleep aid approached significance $(p=.06)$.

Teething. There were also no differences in infant night awakening during the duration of nighttime sleep between infants who were teething (88\%) and those who were not (see Table 10).

Napping. Infants in the sample were taking an average of $2.41(S D=.55)$ naps for an average total of $2.65(S D=.71)$ hours per day. The number of naps the infants were taking in this sample is comparable with the norm for infants this age. Mindell (1997) reported that at 9 months infants take two naps per day.

Infant night awakening was found to be related to napping behavior. Infants who napped more frequently during the day spent more time awake and awoke more frequently during the duration of nighttime sleep than infants who napped less often $(r=.41, p=.01$ and $r=.42, p=$ .01 , respectively). 
Paternal sleep. Paternal average amount of sleep per night were not related to infant average amount of sleep per night $(r=-.14, p=.40)$. The number of times the father awoke during the night was not related to the number of times the infant awoke $(r=.19, p=.24)$ or the amount of time the infant spent awake during the duration of nighttime sleep $(r=.21, p=.21)$. There was no support for the contention that paternal and infant sleep patterns were related to one another in this sample.

\section{Tests of Hypotheses}

Hypothesis 1. Is there a relation between night awakening and infant temperament? It was hypothesized that infants who awoke more during the night would be rated as more difficult by their mothers, cry more, and be "outside the normal limits" on the Behavior Rating Scale more than infants who awoke less during the night. In order to provide support for this hypothesis the total time the infant spent awake and the frequency of infant night awakenings during the duration of nighttime sleep derived from the ICD were correlated with ratings of infant manageability from the RITQ as well as average crying from the ICD, and the overall rating given to the infant on the BRS from the BSID-II by the principal investigator.

Mothers did not rate infants who awoke more frequently and spent more time awake during the duration of nighttime sleep as more difficult (see Table 11). However, the objective measure of infant temperamental difficulty (i.e. crying) was found to be related to infant night awakening (see Table 11). The more an infant cried during the day the more time he or she spent awake and more frequently he or she awakened during the duration of nighttime sleep. A similar pattern of significant correlations was found between the number of times and amount of time the infant cried during the night and the amount of time spent awake and the number of times the infant awakened during the duration of nighttime sleep. Infants who spent more time crying and 
cried more frequently during the night spent more time awake and awakened more frequently during the duration of nighttime sleep.

Finally, the principal investigator's ratings of the infants' behavior during the BSID-II administration and the amount of time the infant spent awake and the frequency of awakenings during the duration of nighttime sleep were not related (see Table 11). Rating the infant’s behavior during the BSID-II as “outside of the normal limits” did not appear to be associated with infant night awakening during the duration of nighttime sleep.

Hypothesis 2. Is infant night awakening related to cognitive performance? It was predicted that infants who spent more time awake and frequently awoke during the night would score lower on the MDI of the BSID-II than infants who awoke less during the night. The infant night awakening variables during the duration of nighttime sleep were correlated with the infants' MDI score to provide support for this hypothesis. Contrary to the proposed hypothesis, infants who more frequently awakened and spent more time awake during the duration of nighttime sleep did not score lower on the MDI $(r=.08, p=.64$ and $r=.04, p=.79$, respectively).

Hypothesis 3. Is maternal sleep deprivation related to perceptions of the infant? It was predicted that mothers who were more sleep deprived would rate their infants as more difficult on the temperament questionnaire, independent of their infant's crying behavior indicated on the ICD and ratings of the infant's behavior during the administration of the BSID-II, than mothers who were less sleep deprived. Partial correlations between the maternal sleep deprivation variable and ratings of infant manageability obtained from the RITQ were calculated while controlling for the infants' crying day and night averages from the ICD and the principal investigator's ratings on the BRS. Partialling of these variables was done in order to assess the 
subjective component of the mother's perception of her infant's temperament independent of the infant's actual behavior. When controlling for the infant's cry total for the night and the principal investigator's BRS rating the partial correlation between the maternal sleep deprivation variable and infant manageability was not significant $(r=.29, p=.08)$. Similarly, when controlling for the infant's cry total for the day and the principal investigator's BRS rating the partial correlation between the maternal sleep deprivation variable and infant manageability was not significant $(r=$ $.05, p=.77)$. Based on these findings it did not appear that mothers who were more sleep deprived perceived their infants as being more difficult.

Hypothesis 4. Is maternal sleep deprivation related to expectations for her infant? It was predicted that mothers who were more sleep deprived would underestimate their infant's performance on the mental items of the BSID-II more than mothers who were less sleep deprived.

In order to find support for this hypothesis, first the number of items the mother reported her infant would pass on the expectation questionnaire and the number of those items the infant actually did pass on the BSID-II was totaled (see box A, Appendix D). Second, the number of items the mother reported her infant would fail and the number of those items the infant actually passed was totaled (see box B, Appendix D). Third, the number of items the mother reported that her infant would pass and the number of those items that the infant actually failed was totaled (see box C, Appendix D). Fourth, the number of items the mother reported that her infant would fail and the number of those items that the infant actually did fail was totaled (see box D, Appendix D).

Maternal overestimation, underestimation, accuracy, and expectations were calculated based on these totals and the total number of items administered from the BSID-II (Karraker, 
Atkins, Coleman, \& Cottrell, 2002). A maternal underestimation variable was calculated by dividing the total number of items the mother reported her infant would fail but that the infant actually passed (B) by the total number of items the infant actually passed $(A+B)$ (see Appendix D). A maternal overestimation variable was calculated by dividing the total number of items the mother reported her infant would pass but that the infant actually failed (C) by the total number of items the infant actually failed (C+D) (see Appendix D). A maternal accuracy variable was calculated by using the proportion of correct expectations the mother had. This variable was calculated by adding the number of items the mother said her infant would pass that the infant did pass (A) and the number of items the mother said her infant would fail that the infant actually did fail (D). This sum was then divided by the total number of items from the item set (see Appendix D). A maternal expectation variable was calculated by adding the total number of items the mother said the infant would pass that the infant did pass (A) and the total number of items the mother said the infant would pass that the infant actually failed (C). This sum was then divided by the total number of items from the item set (see Appendix D). This proportion of items that the mothers expected their infant to pass will be referred to as maternal expectations throughout the remainder of the document. The other variables derived from the proportions described above were used in subsequent analyses. Means, standard deviations, and ranges for these variables are displayed in Table 12.

Finally, to provide support for this hypothesis a correlation between the maternal sleep deprivation variable and the maternal underestimation variable was calculated. Mothers who were more sleep deprived did not appear to be underestimating their infants' performance on the mental items of the BSID-II more than mothers who were less sleep deprived $(r=.20, p=.21)$. 
Hypothesis 5. Is maternal optimism related to maternal perceptions of and expectations for her infant? It was predicted that maternal optimism would be related to more positive perceptions of and higher expectations for her infant. This hypothesis was assessed by correlating the mothers' LOT-R scores with the infant manageability scores obtained from the RITQ and the maternal expectation variable. The findings indicated that in the current sample mothers who were more optimistic did not rate their infants as less difficult $(r=-.12, p=.44)$ and did not have higher expectations for their infant's performance on the mental items of the BSID-II ( $r=-.15, p=.37)$. The findings also indicated that mothers who were more optimistic did not expect their infants to pass more items $(r=-.15, p=.37)$ than mothers who were less optimistic and mothers less optimistic did not expect their infants to fail more times $(r=-.02, p=$ .91) than mothers more optimistic.

Hypothesis 6. Are the effects of sleep deprivation on maternal perceptions of and expectations for her infant moderated by maternal optimism? It was predicted that the mothers' level of optimism would affect the strength of the relation between her level of sleep deprivation and the perceptions and expectations she had for her infant. Specifically, it was hypothesized that the relation between maternal sleep deprivation and perceptions of and expectations for the infant would be weaker when the mother was high in optimism and the relation between maternal sleep deprivation and perceptions of and expectations for the infant would be stronger when the mother was low in optimism.

This hypothesis was tested using the moderation procedure outlined in Baron and Kenny (1986). According to Baron and Kenny, when the moderator is dichotomous (high or low optimism) and the independent variable is continuous (maternal sleep deprivation) then a correlation is computed between maternal sleep deprivation and maternal perceptions of and 
expectations for the infant separately (for high and low optimists) and the difference is tested. A median split was used to divide the sample into high and low optimists and the moderation procedure was performed twice. In the first set of analyses, the independent variable was the maternal sleep deprivation variable, the moderator was the mother's optimism score as measured by the LOT-R, and the dependent variable was the mothers' ratings of their infants' manageability from the RITQ. The correlations between maternal sleep deprivation and infant manageability for high and low optimists were $.18(p=.42)$ and $.15(p=.56)$ respectively. After the tests for moderation were performed a test for the significant differences between the correlations for high and low optimists was computed using the procedure outlined by Bruning and Kintz (1977). This calculation converted the correlation coefficients to z-scores; a significant $z$ indicated that the two correlations were different. The resulting $z$-score for the first set of analyses was .09, indicating that there was no difference between the two correlations.

In the second set of analyses, the independent variable was the maternal sleep deprivation variable, the moderator was the mothers' optimism score as measured by the LOT-R, and the dependent variable was the maternal expectation variable. The correlations for maternal sleep deprivation and maternal expectations for high and low optimists were -.34 $(p=.11)$ and $.17(p=$ .51) respectively. Bruning and Kintz's test for the significant differences between the correlations for high and low optimists was computed again and the resulting z-score for the second set of analyses was .53, indicating that there was no difference between the two correlations. Contrary to the prediction, the relation between maternal sleep deprivation and perceptions of and expectations for the infant was not weaker when the mother was high in optimism and the relation between maternal sleep deprivation and perceptions of and expectations for the infant was not stronger when the mother was low in optimism. 
Hypothesis 7. Are the effects of sleep deprivation on maternal perceptions of and expectations for her infant partially mediated by maternal mood? It was predicted that when the relations between maternal sleep deprivation and mood as well as mood and perceptions of and expectations for her infant were controlled, the relation between maternal sleep deprivation and perceptions of and expectations for her infant would be reduced. According to Baron and Kenny (1986) a variable is considered to be a mediator when it meets three conditions. First, variation in levels of the independent variable must be significantly accounted for by variations in the mediator. Second, variation in levels of the mediator must be significantly accounted for by variation in the dependent variable (Baron \& Kenny). The final condition states that when the first two conditions are controlled for the significant relation between the independent and dependent variables should no longer be significant (Baron \& Kenny). The partial mediation procedure could not be completed in order to test this hypothesis in the present study because the first two conditions were not met (see Table 13).

There were no significant relations between the independent variable (maternal sleep deprivation) and the dependent variables (maternal perception of the infant as measured by the RITQ and maternal expectation variable). There were also no significant relations between the independent variable (maternal sleep deprivation) and the hypothesized mediator (maternal mood as measured by the BDI). Finally, there were no significant relations between the hypothesized mediator (maternal mood) and the dependent variables (maternal perception and maternal expectations). Given that the partial mediation procedure as outlined by Baron and Kenny (1986) could not be conducted there was no support for the hypothesis that the effects of sleep deprivation on maternal perceptions of and expectations for the infant are mediated by the mother's mood in this sample. 


\section{Exploratory Analyses}

Further analyses were conducted to ascertain whether there were any additional significant relations between variables indicated in the figure. The results of these correlational analyses revealed that there were some significant relations among the variables depending on which variable was used to represent the construct depicted in the figure.

Infant dysregulation. As previous research has suggested, there was a significant relation between infant dysregulation and infant night awakening in the present sample. The infant total dysregulation score was calculated with the items pertaining to sleep removed. The results indicated that infants who were rated by their mothers as more dysregulated spent more time awake at night during the duration of nighttime sleep than infants who were rated as less dysregulated $(r=.33, p=.04)$.

Maternal sleep deprivation. Tests of the hypotheses that utilized the continuous maternal sleep deprivation variable were not significant (i.e. hypotheses 3, 4, 6, and 7); therefore additional analyses were conducted using other measures of maternal sleep to ascertain whether any significant relations would result. The analyses for each hypothesis were rerun using maternal average sleep per night, average wake total per night, and wake frequency per night in place of the maternal sleep deprivation variable and no significant associations were found.

In addition to the continuous maternal sleep deprivation another measure of maternal sleep deprivation, maternal ratings of sleep deprivation, was used in several correlational analyses (see Table 14). Mothers were asked to rate their current level of sleep deprivation on the demographic questionnaire using a 5-point scale with 5 being "very sleep deprived”. Maternal ratings of sleep deprivation were found to be related to maternal sleep and wake variables as well as maternal mood and optimism. Mothers whose infants slept less at night rated themselves to be 
more sleep deprived than mothers whose infants slept more at night. Maternal ratings of sleep deprivation on the 5-point scale were also related to maternal mood (as measured by the BDI) and maternal optimism (see Table 14). Mothers who rated themselves as more sleep deprived exhibited more depressive symptoms and were less optimistic.

The actual amount of sleep mothers were receiving was also related to ratings of sleep deprivation and mood (see Table 14). The amount of sleep the mother received the night before the home visit was related to their ratings of sleep deprivation on the 5-point scale and mood on the day of the home visit. Mothers who received less sleep the night before the home visit rated themselves as being more sleep deprived and exhibited more depressive symptoms than mothers who received more sleep the night before the home visit.

BRS ratings. Maternal perceptions of their infant's actual behavior during the BSID-II administration were also of interest in the present study. Specifically, it was predicted that the mothers' perceptions of their infant's actual behavior during the assessment would be different than the principal investigator's observations because past research has indicated that maternal reports of infant behavior tend to be biased. A difference score was computed between mothers’ BRS ratings and the principal investigator's BRS ratings to ascertain how different the two scores were from one another. A one-sample t-test was conducted to determine whether the mean difference score was different from zero. The results revealed that the mean was not different from zero $t(38)=-1.27, p=.21$, indicating that there was no significant difference between the mothers' BRS ratings and the principal investigator's BRS ratings. A correlational analysis was conducted between the mothers' and principal investigator's BRS ratings to assess the strength of the relation between the ratings. The mothers' ratings and the principal investigator's ratings 
were significantly related to one another on two out of the three subscales and the total raw score (see Table 15).

Finally, a t-test was conducted to determine whether there were any differences between mothers who rated their infants’ behavior as more positive than the principal investigator and mothers who rated their infants' behavior as more negative than the principal investigator on the sleep deprivation measure. The t-test indicated that there were no significant differences in maternal sleep deprivation or ratings of maternal sleep deprivation between mothers who rated their infants' behavior as more positive than the principal investigator and mothers who rated their infants' behavior as more negative than the principal investigator $t(37)=-1.18, p=.25, t$ $(37)=.24, p=.81$, respectively.

The joint and differential effects of mood and optimism on maternal perceptions and expectations. The correlational analyses of the variables in Figure 1 revealed that there was a moderate association between maternal mood and optimism (see Table 14). Given that the two variables were moderately correlated with one another it was thought that mood and optimism may be both independently and jointly contributing to maternal perceptions of and expectations for the infant. Therefore, ANOVA analyses were conducted to ascertain the joint and differential effects of maternal mood and optimism on maternal perceptions of and expectations for the infant.

A 2 x 2 ANOVA was conducted to assess the effects of mood and optimism on maternal perceptions of infant manageability. The sample was divided into high and low levels of optimism and high and low levels of depression using median splits. Means and standard deviations as a function of mood and optimism are displayed in Table 16. There was no significant interaction between mood and optimism on ratings of infant manageability $F(1,41)=$ 
$.00, p=1.00$. There were also no significant main effects for optimism $F(1,41)=.09, p=.76$, or mood $F(1,41)=1.13, p=.29$ on ratings of infant manageability. There were no significant differences between individuals who were experiencing high or low levels of optimism and individuals who were experiencing high and low levels of depression in their ratings of infant manageability.

Another 2 x 2 ANOVA was conducted to assess the effects of mood and optimism on maternal expectations for the infant's performance on the cognitive assessment. The sample was divided into high and low levels of optimism and high and low levels of depression using median splits. Means and standard deviations as a function of mood and optimism are displayed in Table 17. There was no significant interaction between mood and optimism on maternal expectations $F$ $(1,41)=.01, p=.92$. There were also no significant main effects for optimism $F(1,41)=.74, p$ $=.39$, or $\operatorname{mood} F(1,41)=.17, p=.68$ on maternal expectations. There were no significant differences between individuals who were experiencing high or low levels of optimism and individuals who were experiencing high and low levels of depression in their expectations for the infant's performance on the cognitive assessment.

Birth status. Finally, it was thought that mothers with more than one child may be receiving less sleep and may be awakened more frequently during the night due to a child other than the infant waking them. Therefore, t-tests were conducted to ascertain whether there were any differences between mothers of first-born and later-born infants on the maternal sleep variables obtained from the ICD. The only significant difference between the mothers was that mothers of later-borns were receiving less sleep than mothers of first-borns. There were no differences between mothers of first-borns and later-borns on maternal wake frequency, wake total, or sleep deprivation (see Table 18). 


\section{Discussion}

The present study sought to determine: 1 ) whether infant characteristics were associated with night awakening and 2) whether variations in infant night awakening were related to infant behavior and maternal characteristics and behaviors. Specific factors were found to be related to infant night awakening in 9-month-olds. Caregiving factors such as feeding method, method used to put the infant to sleep, and co-sleeping practices were all related to infant night awakening. Individual difference factors were also associated with infant night awakening. Infants who experienced separation anxiety, were dysregulated, napped more, and cried more experienced more night awakening than infants who did not have these characteristics. No individual difference factors in the mother were related to maternal perceptions of and expectations for the infant. However, variations in infant night awakening were related to individual differences in maternal characteristics. Mothers whose infants awakened more frequently during the night reported experiencing more sleep deprivation, more depressive symptoms, and lower levels of optimism. Possible explanations for these relations, limitations of the study, and future directions for research in this area are addressed below.

\section{Infant Characteristics Associated with Night Awakening}

Separation anxiety. Mothers’ ratings of infant separation anxiety were one infant characteristic found to be related to infant night awakening. Mothers were asked to indicate whether their infant experienced distress when separated from them. Infants who were experiencing separation anxiety awakened more frequently and spent more time awake at night than infants who were not experiencing separation anxiety. Paret (1983) found a similar result in her study of 9-month-old infants. She speculated that when infants experiencing separation 
anxiety awaken during the night they experience the stress of being separated from their mother and therefore need her presence to help soothe them back to sleep.

The association between separation anxiety and infant night awakening found in the present study also may have occurred because mothers rated their infants' separation anxiety based on how the infant responded to night-time separations. If the infant awakened during the night, signaled to the mother, and cried when the mother attempted to leave the infant to return back to sleep the mother may have interpreted this behavior as separation anxiety. Therefore, in response to the question about whether or not the infant is distressed when separated from her the mother may have responded "yes” based on the way in which the infant behaved upon awakening during the night.

Dysregulation. Another infant characteristic that was related to infant night awakening was dysregulation. Infants who were rated as more dysregulated by their mothers spent more time awake at night. This finding is consistent with past research examining infant dysregulation (DeGangi, 2000). It has been hypothesized that dysregulated infants experience high levels of arousal, which prevents them from inhibiting their alert state to allow themselves to fall into a deep sleep and therefore they spend more time awake during the night (DeGangi).

Temperament. Temperament was one characteristic hypothesized to be related to infant night awakening. There was no support for the hypothesized relation between ratings of infant temperament and infant night awakening. Infants who awoke more frequently during the night were not rated as more difficult by their mothers.

The relation between infant temperament and infant night awakening has mixed support in the literature. Many studies have found an association between temperamental difficulty and infant sleep problems whereas others have not (Morrell \& Steele, 2003). The contention among 
researchers in this area is that when maternal reports of infant temperament are used as indicators of the infant's actual behavior they are not accurate because they are influenced by maternal characteristics (Seifer et al, 1994; Vaughn et al., 1987). Behaviorally assessed temperament has been hypothesized to be more predictive of infant sleep problems than maternal reports of infant temperament (Morrell \& Steele; Scher et al., 1998). This contention was supported by the present study in that infants who cried more frequently during the day spent more time awake and awakened more frequently during the night than infants who cried less during the day. Morrell and Steele (2003) found that fussy-difficult temperament assessed at age 1 accounted for $10 \%$ of the variance in sleep problems at age 1 and was predictive of sleeping problems at age 2 . The authors also found that fussy-difficult temperament influenced the type of strategy the parent used to soothe the child back to sleep. Parents whose infants were fussy-difficult were more likely to physically comfort their infants, which was also predictive of ongoing sleep problems. The authors concluded that the parents were reinforcing the night awakening behavior by physically comforting the infant and were subsequently impeding the development of selfregulation in sleep-wake cycles. Owens-Stively et al. (1997) similarly concluded that parents are more likely to respond to a child who is difficult and easily distressed, therefore reinforcing the night awakenings and resulting in infant sleep problems.

Another explanation for the relation between infant crying during the day and night awakening has been offered by Weissbluth (1989). Weissbluth argued that the sleep deficit that results from frequent night awakenings causes fatigue, which stimulates a stress response in the infant. The subsequent stress response affects the infant's mood, performance, and temperament. As a result the infant becomes more aroused, difficult, and emotional. According to this theory, infants who awoke during the duration of nighttime sleep in the present study may have cried 
more during the day because they were more aroused and emotional due to their lack of sleep. Both the parental reinforcement and infant stress theories are plausible explanations for the relation between infant temperamental difficulty and infant night awakening. Due to the nature of this research it will be impossible to ascertain whether infant temperamental difficulty is causing night awakening or night awakening is causing infant temperamental difficulty due to the fact that random assignment of infants to temperaments is unfeasible.

Finally, infants who awakened more frequently during the night were not behaviorally more difficult during the developmental assessment than infants who awakened less during the night. One reason why there was no relation between infant night awakening and BRS ratings is because the BRS ratings may not have been an adequate indicator of infant temperament. Bayley (1993) stated that the BRS should be used only as an assessment of the infant's behavior during the BSID-II administration. In particular she stated, "behavior tends to be more transient and more susceptible to environmental influences” (p.193). According to Bayley’s contentions, the infant's behavior during the assessment was more of a reflection of the environmental influences (e.g. testing room conditions) they were exposed to rather than their temperament. The lack of a relation between infant night awakening and BRS ratings could also have been due to the low variability in BRS ratings. The principal investigator rated the infants' behavior as within the normal limits for $85 \%$ of the sample. The lack of variability in ratings could have masked the relation between BRS ratings and infant night awakening. Variations in Infant Night Awakening Related to Infant Behavior and Maternal Characteristics and Behavior

Infant behavior. Cognitive performance on the BSID-II was one infant behavior hypothesized to be related to night awakening. However, the results revealed that night 
awakening was not associated with the infants’ cognitive performance. Unlike premature infants, normally developing infants may be spending a sufficient amount of time in quiet sleep even though they may be awakening frequently during the night (Beckwith \& Parmelee, 1986). Given that they are spending enough time in quiet sleep their brain development and organization is not being affected and therefore their cognitive performance would also not be affected (Beckwith \& Parmelee, 1986).

Napping was another infant behavior related to infant night awakening. Infants who napped frequently during the day spent more time awake and awoke more frequently at night than infants who napped less often. This finding is similar to past research and suggests that infants make up for the sleep they miss during the night by taking more naps during the day (Paret, 1983).

Maternal characteristics and behavior. Maternal sleep deprivation that results from infant night awakening was hypothesized to be related to one aspect of maternal behavior, perceptions of the infant. The results revealed that, contrary to the prediction, mothers who were more sleep deprived did not perceive their infants as being more difficult when the infant's actual behavior was statistically controlled. This finding is contrary to past research that found that highly sleep deprived mothers rated their infants as less manageable than not sleep deprived mothers (Karraker \& Cottrell, 2000, Wiedman, 2002). Three-month-old infants were utilized in the previous research that found an association between maternal sleep deprivation and ratings of infant temperament. The mothers may have rated their infants in the present study as more manageable because they were older. It is possible that mothers of 3-month-old infants rated their infants as more difficult because they are still adjusting to the demands of caring for a newborn infant and therefore there may be a greater range of difficulty perceptions at 3 months. 
Mothers of 9-month-olds have already had time to adjust to the demands of caring for the infant and may not rate their infants as difficult as mothers of 3-month-olds. This possibility is likely given that there was little variability in mothers' ratings of infant manageability in the present study. In response to the infant manageability item on the RITQ $82 \%$ of the sample rated their infants on a 6-point scale as 1 being “very easy” or 2 being “easy.” The mothers may have also rated their infants as more positive because they were biased about the behavior of their own children (Sameroff et al. 1982). One factor that has been found to produce that bias is socioeconomic status (Sameroff et al.). Sameroff et al. found that mothers of higher socioeconomic status rated their children as less difficult on the Infant Temperament Questionnaire than mothers of lower socioeconomic status. The mothers in this sample were mainly of higher socioeconomic status, which could account for why they rated their infants as being easy. Another explanation could be that the mothers did not have enough experience with a range of children in order to place their own child on a continuum of temperamental difficulty (Seifer et al. 1994). The RITQ asks the mother "In comparison with other infants you know who are the same age as your infant, how would you rate your infant in the following areas?” (p. 4). Perhaps, the mothers didn't know many infants the same age as their own infant and therefore rated them more positively due to a lack of experience with comparison infants. Another reason why there was little variability in maternal ratings of infant temperament could be attributed to social desirability. Mothers may have rated their infants as less difficult because there is a social pressure placed on mothers to respond positively about their infants. The mother may think she will be perceived as being a bad mother if she were to rate her infant as being difficult, therefore she rates her infant as she thinks she should rather than according to what she actually believes (Shaughnessy, Zechmeister, Zechmeister, 2003). 
Maternal sleep deprivation, resulting from infant night awakening, was also expected to be related to another aspect of maternal behavior, expectations for the infant. Contrary to the proposed hypothesis, mothers who were more sleep deprived did not underestimate their infant's performance on the mental development items more than mothers who were less sleep deprived. Mothers in the present study underestimated $28 \%$ of the items the infant passed. This finding is consistent with past research in samples of mothers whose levels of sleep deprivation were not assessed (Karraker \& Coleman, 1999). Karraker and Coleman found that mothers underestimated $24 \%$ of the mental items on the BSID-II that the infants passed. One explanation for the lack of evidence for the relation between maternal sleep deprivation and expectations could be that sleep deprivation is not a factor that influences maternal expectations. A mother will have expectations about her infant's behavior regardless of the effects of sleep deprivation that she may be experiencing. Miller’s (1988) review of the parental expectation literature suggests that the main factor that influences the relation between parental expectations and children’s cognitive performance is parental knowledge about their child's development. According to this view mothers' expectations about their infants' performance on the cognitive items from the BSID-II in the present study was influenced by her knowledge about her infant's development and not her level of sleep deprivation (Miller; Miller et al. 1991; Stoiber, 1992).

Another maternal characteristic expected to be related to maternal perceptions of and expectations for the infant was maternal optimism. Mothers who were high in optimism were hypothesized as being more positive about their infants than mothers low in optimism. However, in the present sample maternal optimism was not related to perceptions of infant manageability or to maternal expectations. Scheier and Carver $(1985,1992)$ reported that optimists tend to be more realistic than pessimists when confronted with problems and everyday tasks. Given that the 
present sample of mothers was high in optimism they may have rated their infants in a realistic manner and therefore their level of optimism did not inflate their perceptions of and expectations for their infants.

The maternal characteristic of optimism was also expected to moderate the relations between mothers' sleep deprivation and their perceptions of and expectations for their infants. Sleep deprived mothers who were high in optimism were expected to be more accepting of the sleep deprivation they were experiencing as a result of their infant's night awakening than sleep deprived mothers who were low in optimism. However, the results revealed that the relation between maternal sleep deprivation and maternal perceptions of and expectations for the infant was not moderated by optimism. The lack of support for this hypothesis may have been due to the derived maternal sleep deprivation variable. The continuous maternal sleep deprivation variable may not have accurately captured the effects of sleep deprivation. Recall that maternal sleep deprivation was calculated by subtracting the mother's average amount of sleep per night derived from the ICD from the number of hours of sleep she reported that she needs to function well on the background questionnaire. Perhaps some mothers function well on the average amount of sleep they are receiving and therefore they do not experience the effects of sleep deprivation. The maternal rating of sleep deprivation may have been more accurate in its ability to assess the extent to which the mother was experiencing sleep deprivation. When maternal ratings of sleep deprivation were used in the analyses they were related to optimism. Mothers who rated themselves as more sleep deprived were less optimistic. This finding is consistent with past research that has found that parents of infants who frequently awaken during the night encounter problems associated with sleep deprivation such as inability to focus, delayed and poor decision making, indifference, lack of motivation, and high levels of stress (Dement, 1999; 
Wolfson, 1998). Mothers who rated themselves as more sleep deprived in the current study may have been less optimistic because they were experiencing a great deal of stress associated with not receiving enough sleep due to their infants’ night awakening.

Past research has suggested that maternal mood is related to both sleep deprivation and perceptions of the infant (Field, 1995; Foreman \& Henshaw, 2002; Karraker \& Cottrell, 2000; Nover et al., 1984; Wiedman, 2002). Therefore, it was thought that the effects of sleep deprivation on the mother's perceptions of and expectations for her infant could be accounted for by taking into consideration the mother's mood. The partial mediation procedure could not be completed to test this hypothesis because the maternal sleep deprivation variable was not correlated with the hypothesized mediator, maternal mood. The hypothesized mediator also was not correlated with maternal perceptions of and expectations for the infant.

Once again, the lack of evidence for this hypothesis could be attributed to the derived maternal sleep deprivation variable. When maternal ratings of sleep deprivation were used the results revealed that they were related to maternal mood. Mothers who rated themselves as more sleep deprived exhibited more depressive symptoms. The relation between maternal sleep deprivation and maternal mood has been consistently found in the literature. Previous studies have also found that mothers who experience sleep deprivation as a result of their infants’ night awakening report experiencing higher levels of depression feelings and exhibit more depressive symptoms (Cottrell et al. 2002; Goodlin-Jones et al., 1997).

It was surprising that maternal mood and optimism were not related to the total amount of sleep the mothers were receiving or the number of times their sleep was interrupted. Past research that found relations between maternal mood and sleep deprivation utilized maternal night awakenings as an indicator of sleep deprivation (Cottrell et al., 2002; Goodlin-Jones et al., 
1997). In the present study the only variable related to maternal mood and optimism was mothers' own ratings of sleep deprivation. Individual differences in the experience of sleep deprivation may have influenced mothers' ratings of their mood and optimism. For example, two mothers may be receiving the same amount of sleep and may be awakening the same number of times per night; however, one mother rates herself as not sleep deprived and the other mother rates herself as highly sleep deprived. It is likely that the individual variation in how sleep deprived the mother actually feels is accounting for her ratings of mood and optimism.

Finally, the maternal characteristics of mood and optimism were negatively associated with one another. Although a moderate correlation between optimism and depression was apparent, they were not independently or jointly contributing to maternal perceptions of and expectations for the infant. One of the main reasons why the ANOVA analyses may not have been effective in detecting these effects was because of the fact that very few mothers were low in both optimism and depression or high in both optimism and depression. A very large sample would have to be obtained in order to find individuals who are high in both characteristics or low in both characteristics in order to conduct these analyses. Given that mood and optimism are negatively correlated with one another it may be impossible to separate the effects of these variables from one another.

\section{Other Factors Related to Infant Night Awakening}

Several additional factors were hypothesized to be related to infant night awakening in the present study. Previous research examining infant night awakening has suggested that several of the variables were associated with infant night awakening in the second half of the first year.

Feeding method. Infant night awakening was found to be related to feeding method. Infants who were being breast-fed awakened more frequently during the night than infants who 
were bottle-fed. The breast-fed infants may have associated nursing with falling asleep and therefore when they awoke during the night they were unable to soothe themselves back to sleep and required their mother to feed them before they were able to fall back asleep (Burnham et al., 2002; Mindell, 1997). The breast-fed infants may also have awakened more frequently during the night than bottle-fed infants because they may have been hungrier. Breast milk is easier for infants to digest than formula, which means that there has to be a shorter interval between feedings (Mindell, 1997). Therefore, the breast-fed infants may have awakened more frequently to feed and satisfy their hunger.

Method put to sleep. Another variable found to be related to infant night awakening was the method the parent used to put the infant to sleep. Infants who were put to bed asleep spent more time awake at night than infants who were put to bed awake. This finding is consistent with past research that also found that infants who were put to bed asleep spent more time awake during the night and were less able to self-soothe themselves back to sleep (Burnham et al., 2002; Wolfson, 1998). Infants who are put to bed asleep may associate falling asleep with the parent's presence. Therefore, when the infant awakens during the night they have to signal to the parent and be in their presence in order for them to fall back asleep.

Co-sleeping. Infants who co-slept with their mothers awakened more frequently and spent more time awake than infants who did not co-sleep with their mothers. This finding has consistently been reported. Mindell (1997) also found that infants who co-slept with their parents had a harder time falling asleep without their parent's presence, awakened more frequently, and spent less time in the deep stages of sleep than infants who did not co-sleep. Mothers who coslept with their infants may also have been more likely to report infant night awakening. Mothers who co-slept with their infants in the present study may have been more likely to report infant 
night awakening because they were more likely to be awakened when their infant awoke, whereas mothers who did not co-sleep would not have reported a night awakening unless their infant signaled them.

McKenna et al. (1994) found in their co-sleeping study that mothers and infants who cosleep show overlapping night awakening patterns, which are induced by the co-sleeping partner. McKenna et al. found that one factor that accounted for these overlapping night awakenings is the amount of direct physical contact the mother and the infant have while they are sleeping. If one co-sleeping partner makes a sudden move or sound then it is likely to awaken the other partner. The authors argued that this frequent night awakening is beneficial to the infant because it reduces the likelihood that the infant will suffer from Sudden Infant Death Syndrome (SIDS). All researchers who study co-sleeping do not hold the contention that co-sleeping is beneficial to the infant. Hunsley and Thoman (2002) found in their study of co-sleeping that infants who coslept showed less quiet sleep, more arousals, and more sleep-stage shifts than infants who did not co-sleep. This sleeping pattern is consistent with sleep fragmentation and has been found to be associated with increases in illness, poor performance on cognitive assessments, and other negative developmental outcomes in infants. Based on their findings, the authors concluded that co-sleeping might have detrimental effects on the infant's neurobehavioral development. This collection of findings suggests that although co-sleeping may be beneficial to the infant in the short-term given that it may prevent SIDS it may not be beneficial to the infant in the long-term because the infant may experience developmental lags

Teething. No differences were found between infants who were teething and infants who were not teething in the amount of time they spent awake at night or the number of times they 
awakened during the night. Similar to past research the pain of teething did not appear to be associated with infant night awakening (Paret, 1983).

Use of a sleep aid. Another surprising finding was that there were no differences in infant night awakening between infants who used a sleep aid (i.e. pacifier, blanket, stuffed animal) and infants who did not use a sleep aid, although this difference did approach significance in the present study. Past research has shown that infants who do not use a sleep aid awaken more frequently than infants who do use a sleep aid (Mindell, 1997; Paret, 1983). Paret found that that the use of a sleep aid was related to sleeping through the night. Infants who use a sleep aid may be able to soothe themselves back to sleep by utilizing their sleep aid instead of having to signal to their parents. Infants who do not use a sleep aid may not be able to soothe themselves back to sleep because they do not have an immediate source of comfort and therefore may need to signal to their parents to receive help in soothing them back to sleep. Further research using a larger sample size needs to be conducted to ascertain whether there is a relation between sleep aid use and infant night awakening.

Paternal night awakening. Infant night awakening was not related to paternal night awakening. However, one limitation of this finding is that maternal reports of their partner's night awakening were relied upon to measure paternal night awakening. It is possible that the mothers may not have been accurate in the accounts of fathers' sleeping and waking behaviors, especially if the mother did not awaken when her partner did. A more accurate measure of paternal sleep and night awakening could have been obtained by having the father complete a sleep diary as well. Further research should be conducted, using a paternal sleep diary, in order to better ascertain whether there is a genetic component related to infant night awakening. 
Birth status. Finally, mothers with more than one child were expected to be receiving less sleep and being awakened more frequently due to a child other than the infant waking her. Mothers of later-borns were receiving less sleep than mothers of first-borns. However, they were not being awakened more frequently, did not spend more time awake during the night, and were not more sleep deprived than mothers of first-borns. These findings suggest that having more than one child may not influence the frequency of night awakenings and the amount of time spent awake at night but may influence the amount of sleep the mother is receiving. Perhaps mothers of more than one child are receiving less sleep because they cannot go to sleep when the infant does, like mothers of first-borns can, given that they have to attend to older children. Another reason why mothers of later-borns were receiving less sleep may be because they had to get up earlier to attend to the older children. Therefore, mothers of later-borns may be receiving less sleep because they cannot sleep until the older children are asleep and may have to wake up earlier to perform tasks necessary to take care of multiple children.

\section{Maternal Perceptions of Infant Behavior}

An additional interest in the present study was maternal perceptions of infant behavior after the developmental assessment because past research has suggested that parents are not accurate in rating the behavior of their own children (Seifer et al. 1994). Therefore, mothers' ratings of their infants' behavior as measured by the BRS were compared to the principal investigator's ratings of the infants' behavior on the BRS.

Maternal BRS ratings were not different than the principal investigator’s BRS ratings. Although these findings are contrary to the prediction, they indicate that mothers' perceptions of their infants' behavior during a developmental assessment were not systematically distorted and can be used as an additional source of information about the infant's behavior. Although the 
mothers' BRS ratings were not different on average from the principal investigator's BRS ratings, the correlational analyses revealed that the ratings were only related on two out of the three subscales. Maternal ratings and the principal investigator's ratings on the motor scale were not related to one another. This finding could be attributed to the directions given to the mothers before the task. The principal investigator instructed the mother to rate her infant's behavior during the assessment and to ask questions if any of the items needed clarification. Very few mothers asked for clarification and it is possible that the mothers did not understand some of the items. Future researchers who choose to use the BRS with caregivers should provide mothers with descriptions of each BRS item from the BSID-II manual to help provide clarification.

\section{Limitations and Future Directions}

Several general limitations of the study should be acknowledged. First, the sample was mainly composed of women from high socioeconomic backgrounds who were highly optimistic and therefore the results may not be generalizable to the population. Another limitation of the study was that an examination of the causal mechanisms underlying infant night awakening could not be directly tested. Many of the findings raise questions about causality. For example, was infant night awakening related to infant crying because the infant's night awakening was causing them to be difficult or because their temperamental difficulty was causing them to awaken during the night? Also, were maternal ratings of sleep deprivation related to higher depressive symptoms because the mother was not receiving enough sleep or was the mother not receiving enough sleep because she was experiencing the depressive symptoms? Due to the nature of this research it is impossible to assess these causal relations because random assignment of mothers and infants to temperaments, moods, and sleeping patterns is not possible. The findings of the present study can inform future research, which could recruit larger numbers 
of mother-infant dyads and utilize statistical techniques to test the causal links among the relations suggested in the figure. The results of studies utilizing structural equation modeling or path analysis could provide a better idea of which factors are causing infant night awakening.

The findings of the present study can also be used to guide future research, which could utilize experimental methods to ascertain whether infant night awakening can be reduced or eliminated by manipulating environmental conditions. There is a body of literature on attempts to reduce children’s sleep problems by utilizing experimental research methods. Wolfson’s (1998) review of this research indicated that the majority of the research programs teach parents behavior management strategies that attempt to decrease children's night awakening. However, the majority of the research cited was conducted using parents seeking some type of clinical treatment for their child's sleep problems. This may be a highly selective group of children who have been experiencing severe sleep problems for years. Few studies have attempted to intervene with parents of young infants (Wolfson, 1998). The findings of the present study can inform this research, which could manipulate certain environmental conditions to reduce or eliminate infant night awakening. Past research has shown that introducing a sleep aid to the infant is related to maternal reports of well-being (Burnham et al. 2002; Goodlin-Jones et al., 1997). Mothers whose infants utilized a sleep aid reported feeling less depressed (Goodlin-Jones et al., 1997). One limitation of this research is that the sleep aid group was not compared to a group of infants who did not use a sleep aid. Future research could build on this methodology and randomly assign infants to a sleep aid condition or a control condition with no sleep aid to ascertain whether infants in the sleep aid condition utilize their sleep aid to soothe themselves back to sleep upon awakening rather than signaling to their parents. If night awakening is reduced through these 
experimental methods then the difficulties associated with maternal sleep deprivation, such as deficits in mood and optimism, could also be alleviated.

\section{Conclusion}

The present study was able to identify infant and maternal characteristics and behaviors related to infant night awakening in 9-month-olds. The variables examined in the study have been independently examined by various other researchers but have never been examined together in a sample of healthy, normally developing 9-month-old infants. The study was unique because it explored relations among variables such as maternal sleep deprivation, mood, optimism, and expectations of and perceptions for the infant that had yet to be explored in the literature. The findings indicate that individual difference factors between infants are related to night awakening and that variations in infant night awakening are related to maternal sleep deprivation, mood, and optimism. The findings of this research can be used to inform experimental research, which can target specific infant and environmental characteristics to reduce infant night awakening and alleviate the effects of sleep deprivation of parents attending to their waking infants. 


\section{References}

Anders, T. (1979). Night-waking in infants during the first year of life. Pediatrics, 63, 860-864.

Anders, T. (1994). Infant sleep, nighttime relations, and attachment. Psychiatry, 57, 11-21.

Anders, T. \& Keener, M. (1985). Developmental course of nighttime sleep-wake patterns in fullterm and premature infants during the first year of life. I. Sleep, 8, 173-192.

Anders, T., Keener, M., Bowe, T., Shoaff, B. (1983). A longitudinal study of nighttime sleepwake patterns in infants from birth to one year. In J. Call, E. Glaneson, \& R. Tyson (Eds.), Frontiers in Infant Psychiatry (pp.150-166). New York: Basic Books.

Bamford, F., Bannister, R., Benjamin, C., Hillier, V., Ward, B., \& Moore, W. (1990). Sleep in the first year of life. Developmental Medicine \& Child Neurobiology, 32, 718-724.

Baron, R., \& Kenny, D. (1986). The moderator-mediator variable distinction in social psychological research: Conceptual, strategic, and statistical considerations. Journal of Personality and Social Psychology, 51, 1173-1182.

Bates, J. (1980). The concept of difficult temperament. Merrill-Palmer Quarterly, 26, 299-319.

Bates, J. \& Bayles, K. (1984). Objective and subjective components in mother's perceptions of their children from age 6 months to 3 years. Merrill-Palmer Quarterly, 30, 111-130.

Bates, J., Viken, R., Alexander, D., Beyers, J., \& Stockton, L. (2002). Sleep and adjustment in preschool children: Sleep diary reports by mothers relate to behavior reports by teachers. Child Development, 73, 62-74.

Bayley, N. (1993). Bayley scales of infant development: Second edition. San Antonio: The Psychological Corporation.

Beck, A., Steer, R., \& Garbin, M. (1988). Psychometric properties of the Beck Depression Inventory: Twenty-five years of evaluation. Clinical Psychology Review, 8, 77-180. 
Beckwith, L., \& Parmelee, A. (1986). EEG patterns of preterm infants, home environment, and later IQ. Child Development, 57, 777-789.

Belsky, J. (1984). The determinants of parenting: A process model. Child Development, 55, 8396.

Belsky, J., Crnic, K., \& Woodworth, S. (1995). Personality and parenting: Exploring the mediating role of transient mood and daily hassles. Journal of Personality, 63, 905-929.

Bonnet, M. (1989). Infrequent periodic sleep disruption: Effects on sleep, performance, and mood. Physiology \& Behavior, 45, 1049-1055.

Bruning, J.L., \& Kintz, B.L. (Eds.). (1977). Computational handbook of statistics (2 ${ }^{\text {nd }}$ ed.). Glenview, IL: Scott, Foresman, and Company.

Burnham, M., Goodlin-Jones, B., Gaylor, E., \& Anders, T. (2002). Nighttime sleep-wake patterns and self-soothing from birth to one year of age: A longitudinal intervention study. Journal of Child Psychology and Psychiatry and Allied Disciplines, 43, 713-725.

Carey Temperament Scales: Test Manual. (1996). Scottsdale, AZ: Behavioral-Development Initiatives.

Carey, W. (1974). Night waking and temperament in infancy. Behavioral Pediatrics, 84, 756758.

Clark, L., Kochanska, G., \& Ready, R. (2000). Mothers’ personality and its interaction with child temperament as predictors of parenting behavior. Journal of Personality and Social Psychology, 79, 274-285.

Cottrell, L., Atkins, M., \& Khan, A. (2002). The impact of infant epilepsy on maternal sleep disruption and socio-emotional functioning. Poster presented at the International Conference on Infant Studies, Toronto, CA. 
Crowe, T., Clark, L., \& Qualls, C. (1996). The impact of child characteristics on mothers' sleep patterns. The Occupational Therapy Journal of Research, 16, 3-22.

DeGangi, G. (2000). Pediatric disorders of regulation in affect and behavior. New York: Academic Press.

DeGangi, G., Poisson, S., Sickel, R., \& Wiener, A. S. (1995). Infant/Toddler Symptom Checklist: A screening tool for parents. Tucson, AZ: Therapy Skill Builders.

Dement, W. (1999). The promise of sleep. New York: Delacorte Press.

DiPietro, J., \& Porges, S. (1991). Relations between neonatal states and 8-month developmental outcome in preterm infants. Infant Behavior and Development, 14, 441-450.

Ferber, R. (1985). Sleep, sleepiness, and sleep disturbances in infants and young children. Annals of Clinical Research, 17, 227-234.

Field, T. (1995). Infants of depressed mothers. Infant Behavior and Development, 18, 1-13.

Foreman, D., \& Henshaw, C. (2002). Objectivity and subjectivity in postnatally depressed mothers’ perceptions of their infants. Child Psychiatry and Human Development, 32, 263-275.

Goodlin-Jones, B., Eiben, L. \& Anders, T. (1997). Maternal well-being and sleep-wake behaviors in infants: An intervention using maternal odor. Infant Mental Health Journal, 18, 378-393.

Halpern, L., \& McLean, W. (1997). Hey mom, look at me. Infant Behavior and Development, 20, 515-529.

Hunsley, M., \& Thoman, E.B. (2002). The sleep of co-sleeping infants when they are not cosleeping: Evidence that co-sleeping is stressful. Developmental Psychobiology, 40, 14-22. 
Hunt, J. \& Paraskevopoulos, J. (1980). Children’s psychological development as a function of the inaccuracy of their mothers’ knowledge of their abilities. The Journal of Genetic Psychology, 136, 285-298.

Jones, D., Forehand, R., Brody, G., \& Armistead, L. (2002). Positive parenting and child psychosocial adjustment in inner-city single-parent African American families. Behavior Modification, 26, 464-481.

Karraker, K.H., Atkins, M., Coleman, P., \& Cottrell, L. (2002, April). Mothers’ expectations for their infants’ performance on the Bayley Scales of Infant Development. In M. Stern (Chair), Maternal expectations, self-efficacy, and other cognitions: Impact on caregiving and infant outcomes. Symposium conducted at the meeting of the International Conference on Infant Studies, Toronto, Canada.

Karraker, K.H., \& Coleman, P. (1999, April). Mothers' predictions of their toddlers' performance on the Bayley Scales of Infant Development. Poster presented at the Society for Research in Child Development, Albuquerque, NM.

Karraker, K.H. \& Cottrell, L. (2000, July). Impact of sleep deprivation on socioemotional functioning of mothers and young infants. Poster presented at the International Conference on Infant Studies, Brighton, UK.

Karraker, K.H., \& Evans, S.L. (1995). Adolescent mothers’ knowledge of child development and expectations for their own infants, Journal of Youth and Adolescence, 25, 651-665.

Kochanska, G., Clark, L., \& Goldman, M. (1997). Implications of mothers’ personality for their parenting and their young children’s developmental outcomes. Journal of Personality, 65, 387-420. 
Lounsbury, M., \& Bates, J. (1982). The cries of infants of differing levels of perceived temperamental difficultness: Acoustic properties and effects on listeners. Child Development, 53, 677-686.

McKenna, J., Mosko, S., Richard, C., Drummond, S., Hunt, L., Cetel, M., \& Arpaia, J. (1994). Experimental studies of infant-parent co-sleeping: Mutual physiological and behavioral influences and their relevance to SIDS. Early Human Development, 38, 187-201.

Miller, S. (1988). Parents’ beliefs about children’s cognitive development. Child Development, 59, 259-285.

Miller, S., Manhal, M., \& Mee, L. (1991). Parental beliefs, parental accuracy, and children’s cognitive performance: A search for causal relations. Developmental Psychology, 27, 267-276.

Mindell, J. (1997). Sleep through the night. New York: HarperCollins Publishers.

Morrell, J. \& Steele, H. (2003). The role of attachment security, temperament, maternal perception, and care-giving behavior in persistent infant sleeping problems. Infant Mental Health Journal, 24, 447-468.

Nover, A., Shore, M., Timberlake, E. \& Greenspan, S. (1984). The relation of maternal perception and maternal behavior: a study of normal mothers and their infants. American Journal of Orthopsychiatry, 54, 210-223.

Owens-Stively, J., Frank, N., Smith, A., Hagino, O., Spirito, A., Arrigan, M., \& Alario, A. (1997). Child temperament, parenting discipline style, and daytime behavior in childhood sleep disorders. Developmental and Behavioral Pediatrics, 18, 314-321. 
Paret, I. (1983). Night waking and its relation to mother-infant interaction in 9-month-olds. In J.

Call, E. Glaneson, and R. Tyson (Eds.). Frontiers of Infant Psychiatry (pp.171-177). New York: Basic Books.

Peterson, C. (2000). The future of optimism. American Psychologist, 55, 44-55.

Pilcher, J. \& Huffcutt, A. (1996). Effects of sleep deprivation on performance: A meta-analysis. Sleep, 19, 318-326.

Sameroff, A., Seifer, R., \& Elias, P. (1982). Sociocultural variability in infant temperament ratings. Child Development, 53, 164-173.

Santrock, J.W. (2002). A topical approach to life-span human development. Boston: McGraw Hill Publishers.

Scher, A., Epstein, R., Sadeh, A., Tirosh, E., \& Lavie, P. (1992). Toddlers’ sleep and temperament: Reporting bias or a valid link? A research note. Journal of Child Psychology and Psychiatry, 33, 1249-1254.

Scher, A., Tirosh, E., \& Lavie, P. (1998). The relation between sleep and temperament revisited: Evidence for 12-month-olds. A research note. Journal of Child Psychology and Psychiatry, 39, 785-788.

Scheier, M., \& Carver, C. (1985). Optimism, coping, and health: Assessment and implications of generalized outcome expectancies. Health Psychology, 4, 219-247.

Scheier, M., \& Carver, C. (1992). Effects of optimism on psychological and physical well-being: Theoretical overview and empirical update. Cognitive Therapy and Research, 16, 201228. 
Scheier, M., Carver, C., \& Bridges, M. (1994). Distinguishing optimism from neuroticism (and trait anxiety, self-mastery, and self-esteem): A reevaluation of the Life Orientation Test. Journal of Personality and Social Psychology, 67, 1063-1078.

Shaughnessy, J.J., Zechmeister, E.B., \& Zechmeister J.S. (2003). Research methods in psychology. (6 ${ }^{\text {th }}$ ed.). New York, NY; McGraw-Hill Higher Education.

Seifer, R., Sameroff, A., Barrett, L., \& Krafchuk, E. (1994). Infant temperament measured by multiple observations and mother report. Child Development, 65, 1478-1490.

Stoiber, K. (1992). Parents’ beliefs about their children's cognitive, social, and motor functioning. Early Education and Development, 3, 244-259.

Stoiber, K. \& Houghton, T. (1993). The relation of adolescent mothers’ expectations, knowledge, and beliefs to their young children's coping behavior. Infant Mental Health Journal, 14, 61-79.

Tamis-LeMonda, C., Chen, L., \& Bornstein, M. (1998). Mothers’ knowledge about children’s play and language development: Short-term stability and interrelations. Developmental Psychology, 34, 115-124.

Thoman, E. (2001). Sleep-wake states as context for assessment, as components of assessment, and as assessment. In L. Singer \& P. Zeskind (Eds.), Biobehavioral assessment of the infant (pp.125-145). New York: The Guilford Press.

Tollison, P., Palmer, D.J., \& Stow, M.L. (1987). Mothers’ expectations, interactions, and achievement attributions for their learning disabled or normally achieving sons. The Journal of Special Education, 21, 83-93. 
Van Tassel, E. (1985). The relative influence of child and environmental characteristics on sleep disturbances in the first and second years of life. Developmental and Behavioral Pediatrics, 6, 81-86.

Vaughn, B., Bradley, C., Joff, L., Seifer, R., \& Barglow, P. (1987). Maternal characteristics measured prenatally are predictive ratings of temperamental difficulty on the Carey Infant Temperament Questionnaire. Developmental Psychology, 23, 152-161.

Weissbluth, M. (1989). Sleep-loss stress and temperamental difficultness: Psychobiological processes and practical considerations. In G. Kohnstamm, J. Bates, \& M. Rothbart (Eds.), Temperament in childhood (pp.357-375). New York: John Wiley and Sons.

Wiedman, C. (2002). The effects of maternal sleep deprivation on maternal sensitivity and contingent responsiveness. Unpublished master’s thesis. West Virginia University.

Wolfson, A. (1998). Working with parents on developing efficacious sleep/wake habits for infants and young children. In J. Briesmeister, and C. Schaefer (Eds.). Handbook of parent training: Parents as co-therapists for children's behavior problems ( $2^{\text {nd }}$ Edition) (pp.347-383). New York: John Wiley and Sons.

Zuckerman, B., Stevenson, J., \& Bailey, V. (1987). Sleep problems in early childhood: Continuities, predictive factors, and behavioral correlates. Pediatrics, 80, 664-671. 
Table 1

Participant Characteristics

Characteristic

Race

Non-Hispanic white

Asian

Other

Marital status

Married

100

Single

Employment status

Full time

Part time

Volunteer

Homemaker

Education

High school degree

Some college

4-year college degree

Some graduate school

Graduate degree
29

2

$\%$ 


\section{Characteristic}

Infant birth status

First-born

Later-born

Day care attendance

Attended day care

34

Did not attend day care
$\%$

42

56 
Table 2

Participant Demographic Variables

\begin{tabular}{lccc}
\multicolumn{1}{l}{ Measure } & Range & $M$ & $S D$ \\
\hline Maternal age & $23-40$ & 31.08 & 4.37 \\
Income & $10,000-200,000$ & 69,341 & 44,967 \\
\end{tabular}

Note. Means, ranges, and standard deviations for the variables listed in the table were computed in years and dollars, respectively. 
Table 3

Means, Standard Deviations, and Ranges for Variables in Figure 1

\begin{tabular}{lccc}
\hline \multicolumn{1}{c}{ Measure } & Range & $M$ & \\
& & & \\
\hline ITSC & $0-27$ & 7.34 & 6.75 \\
MDI & $82-111$ & 97.93 & 6.47 \\
ITQ manageability & $1-6$ & 1.85 & 1.13 \\
Maternal sleep deprivation & $-3.36-2.04$ & $-3.98 \mathrm{E}-02$ & 1.19 \\
BDI-II & $0-31$ & 7.76 & 7.22 \\
LOT-R & $7-24$ & 17.00 & 4.30 \\
\hline
\end{tabular}

Note. ITSC = Infant Toddler Symptom Checklist score; MDI = Mental Development Index; BDI

= Total score on the Beck Depression Inventory-II; ITQ = Infant Temperament Questionnaire;

LOT-R = Total score on the Life Orientation Test Revised. 
Table 4

Participant Means, Standard Deviations, and Ranges from the Infant Care Diary

\begin{tabular}{|c|c|c|c|}
\hline Measure & Range & $M$ & $S D$ \\
\hline Mom nap total & $0-2.64$ & .26 & .48 \\
\hline Mom nap frequency & $0-1.43$ & .21 & .29 \\
\hline Mom sleep total & 3.61-9.36 & 6.83 & 1.07 \\
\hline Mom wake total & $0-2.07$ & .77 & .56 \\
\hline Mom wake frequency & $0-3.86$ & 1.55 & 1.03 \\
\hline Infant nap total & $1.18-4.18$ & 2.65 & .71 \\
\hline Infant nap frequency & $1.14-3.43$ & 2.41 & .55 \\
\hline Infant sleep total & 8.14-12.11 & 9.77 & .89 \\
\hline Infant wake total & $0-2.07$ & .60 & .46 \\
\hline \multicolumn{4}{|l|}{ during duration of } \\
\hline \multicolumn{4}{|l|}{ nighttime sleep } \\
\hline Infant wake frequency & $0-3.57$ & 1.29 & 1.00 \\
\hline \multicolumn{4}{|l|}{ during duration of } \\
\hline \multicolumn{4}{|l|}{ nighttime sleep } \\
\hline Infant wake total & $0-1.14$ & .42 & .35 \\
\hline \multicolumn{4}{|l|}{ during maternal } \\
\hline sleep & & & \\
\hline
\end{tabular}




\begin{tabular}{lccc}
\hline \multicolumn{1}{c}{ Measure } & Range & $M$ & $S D$ \\
\hline Infant wake frequency & $0-2.86$ & 1.00 & .82 \\
during maternal & & & .96 \\
sleep & $0-4.11$ & 1.06 & 2.68 \\
Infant cry total day & $0-11.14$ & 3.26 & .26 \\
Infant cry frequency & & & .85 \\
day & $0-.89$ & .95 & \\
Infant cry total night & $0-3.00$ & & \\
Infant cry frequency & &
\end{tabular}

Note. Means, ranges, and standard deviations for the variables listed in the table were computed in hours per day. 
Table 5

Night Awakening Differences During the Duration of Nighttime Sleep Between Infants Who Breast-Feed and Infants Who Bottle-Feed

$\underline{\text { Breast-fed } \quad \text { Bottle-Fed }}$

\begin{tabular}{lccccccc} 
Night awakening variable & $M$ & $S D$ & $n$ & $M$ & $S D$ & $n$ & $t(32)$ \\
\hline Infant wake frequency & 1.91 & 1.02 & 15 & .99 & .85 & 19 & $2.90 * *$ \\
Infant wake total & .81 & .51 & & .52 & .43 & & 1.82 \\
\end{tabular}

$* * p<.01$ 
Table 6

Night Awakening Differences During the Duration of Nighttime Sleep Between Infants Who Are Put To Bed Awake and Infants Who Are Put To Bed Asleep

\begin{tabular}{lccccccc} 
& \multicolumn{3}{c}{ Awake } & & \multicolumn{3}{c}{ Asleep } \\
Night awakening variable & $M$ & $S D$ & $n$ & $M$ & $S D$ & $n$ & $t(39)$ \\
\hline Infant wake frequency & 1.08 & .97 & 23 & 1.56 & 1.01 & 18 & -1.56 \\
Infant wake total & .39 & .32 & & .86 & .49 & & $-3.63^{* *}$ \\
\hline$* * p<.01$.
\end{tabular}


Table 7

Night Awakening Differences During the Duration of Nighttime Sleep Between Infants Who Cosleep and Infants Who Do Not Co-sleep

\section{Co-sleep $\quad$ Do not co-sleep}

\begin{tabular}{lccccccc} 
Night awakening variable & $M$ & $S D$ & $n$ & $M$ & $S D$ & $n$ & $t(39)$ \\
\hline Infant wake frequency & 2.04 & .96 & 10 & 1.05 & .91 & 31 & $2.97 * *$ \\
Infant wake total & 1.03 & .43 & & .46 & .38 & & $4.00^{* *}$
\end{tabular}

$* * p<.01$ 
Table 8

Night Awakening Differences During the Duration of Nighttime Sleep Between Infants with Separation Anxiety and Infants without Separation Anxiety

\section{Separation anxiety $\quad$ No separation anxiety}

\begin{tabular}{lccccccc} 
Night awakening variable & $M$ & $S D$ & $n$ & $M$ & $S D$ & $n$ & $t(39)$ \\
\hline Infant wake frequency & 1.58 & 1.06 & 25 & .85 & .73 & 16 & $2.40^{*}$ \\
Infant wake total & .73 & .49 & & .38 & .33 & & $2.56^{*}$ \\
& & & & & & &
\end{tabular}


Table 9

Night Awakening Differences During the Duration of Nighttime Sleep Between Infants Who Use a Sleep Aid and Infants Who Do Not Use a Sleep Aid

\begin{tabular}{lcccccccc} 
& \multicolumn{3}{c}{ Sleep Aid } & \multicolumn{5}{c}{ No Sleep Aid } \\
\multicolumn{1}{c}{ Night awakening variable } & $M$ & $S D$ & $n$ & $M$ & $S D$ & $n$ & $t(39)$ \\
\hline Infant wake frequency & 1.08 & .73 & 27 & 1.70 & 1.02 & 14 & -1.96 \\
Infant wake total & .52 & .48 & & .74 & .40 & & -1.43 \\
\hline
\end{tabular}


Table 10

Night Awakening Differences During the Duration of Nighttime Sleep Between Infants Who were Teething and Infants Who were Not Teething

Teething

\begin{tabular}{lccccccc} 
Night awakening variable & $M$ & $S D$ & $n$ & $M$ & $S D$ & $n$ & $t(39)$ \\
\hline Infant wake frequency & 1.36 & 1.05 & 36 & .80 & .41 & 5 & 1.18 \\
Infant wake total & .61 & .48 & & .48 & .36 & .61 \\
\hline
\end{tabular}


Table 11

Intercorrelations Between Infant Night Awakening During the Duration of Nighttime Sleep and Infant Temperament and Behavior

\begin{tabular}{lcc}
\multicolumn{1}{r}{ Measure } & Wake total & Wake frequency \\
\hline ITQ manageability & .10 & .08 \\
Cry total day & .27 & $.33^{*}$ \\
Cry frequency day & $.36^{*}$ & $.53^{* * *}$ \\
Cry total night & $.63^{* * *}$ & $.66^{* * *}$ \\
Cry frequency night & $.66^{* * *}$ & .08 \\
PI BRS & .00 &
\end{tabular}

Note. ITQ = Infant Temperament Questionnaire subscale; PI BRS = Principal investigator’s

Behavior Rating Scale total rating score.

${ }^{*} p<.05 . * * * p<.001$. 
Table 12

Means, Standard Deviations, and Ranges for the Maternal Expectation Variables

\begin{tabular}{lccc}
\hline \multicolumn{1}{c}{ Measure } & Range & $M$ & \\
& & & $S D$ \\
\hline Accuracy & $.36-.84$ & .60 & .10 \\
Expectation & $.36-.88$ & .63 & .11 \\
Underestimation & $.06-.50$ & .28 & .12 \\
Overestimation & $.15-.86$ & .52 & .17 \\
\hline
\end{tabular}


Table 13

Intercorrelations Between Maternal Perceptions of and Expectations for the Infant and Maternal Characteristics

\begin{tabular}{|c|c|c|c|c|}
\hline Measure & 1 & 2 & 3 & 4 \\
\hline $\begin{array}{l}\text { 1. Maternal perception of } \\
\text { infant manageability }\end{array}$ & - & .16 & .22 & .03 \\
\hline 2. Maternal sleep deprivation & & - & -.04 & -.05 \\
\hline 3. BDI-II & & & - & .07 \\
\hline 4. Maternal expectation & & & & - \\
\hline
\end{tabular}

Note. BDI-II = Total score on the Beck Depression Inventory-II. 
Table 14

Intercorrelations Between Infant Sleep Variables and Maternal Sleep Variables and Characteristics

\begin{tabular}{|c|c|c|c|c|c|c|c|c|c|}
\hline Measure & 1 & 2 & 3 & 4 & 5 & 6 & 7 & 8 & 9 \\
\hline 1. Average sleep & - & $.70 * * *$ & $.56 * * *$ & $.71 * * *$ & .16 & $-.60 * * *$ & -.30 & $-.31 *$ & $.66 * * *$ \\
\hline 2. Sleep function & $.70 * * *$ & - & $.65 * * *$ & $.37 *$ & .30 & -.22 & -.19 & $-.36^{*}$ & $.49 * *$ \\
\hline 3. Ideal sleep & $.56 * * *$ & $.65 * * *$ & - & .19 & .25 & -.07 & .03 & -.28 & $.34 *$ \\
\hline 4. Sleep last night & $.71 * * *$ & $.37^{*}$ & .19 & - & -.05 & $-.74 * * *$ & $-.49 * * *$ & .02 & $.58 * * *$ \\
\hline 5. Sleep important & .16 & .30 & .25 & -.05 & - & .05 & -.02 & $-.34 *$ & .16 \\
\hline 6. Rate SD & $-.60 * * *$ & -.22 & -.07 & $-.74 * * *$ & .05 & - & $.38 *$ & .26 & $-.61 * * *$ \\
\hline 7. ICD MNF & -.30 & -.19 & .03 & $-.48^{* *}$ & -.02 & .38 & - & -.21 & $-.41 * *$ \\
\hline 8. ICD MWF & $-.31 *$ & $-.36 *$ & -.28 & .02 & $-.34 *$ & .26 & -.21 & - & $-.38 *$ \\
\hline 9. ICD MST & $.66 * * *$ & $.49 * *$ & $.34 *$ & $.59 * * *$ & .16 & $-.62 * * *$ & $-.41 * *$ & $-.37 *$ & - \\
\hline 10. Maternal SD & .15 & $.63 * * *$ & .39 & -.12 & .18 & .31 & .16 & -.05 & $-.37 *$ \\
\hline 11. ICD MWT & $-.40 *$ & $-.45^{* *}$ & -.26 & -.17 & -.34 & $.39 *$ & .02 & $.78 * *$ & $-.43^{* *}$ \\
\hline 12. ICD MNT & -.25 & -.20 & -.01 & $-.46^{* *}$ & .04 & $.32 *$ & $.93 * * *$ & -.25 & $-.43 * *$ \\
\hline 13. ICD BWT & $-.35 *$ & -.29 & -.30 & -.14 & -.23 & .24 & -.03 & $.63^{* * *}$ & -.23 \\
\hline 14. ICD BWF & -.29 & -.22 & $-.33^{*}$ & .00 & -.29 & .19 & -.12 & $.71 * * *$ & -.14 \\
\hline 15. ICD BST & $.36^{*}$ & $.33 *$ & .30 & .12 & .29 & $-.32 *$ & -.21 & $-.48 * *$ & $.47^{* *}$ \\
\hline 16. BDI-II & -.20 & -.09 & .17 & $-.37 *$ & -.02 & .36 & .11 & -.04 & -.07 \\
\hline 17. LOT-R & .22 & .04 & -.04 & .28 & -.16 & -.32 & .02 & .14 & .00 \\
\hline
\end{tabular}




\begin{tabular}{|c|c|c|c|c|c|c|c|c|}
\hline Measure & 10 & 11 & 12 & 13 & 14 & 15 & 16 & 17 \\
\hline 1. Average sleep & .15 & $-.40^{*}$ & -.25 & $-.35 *$ & -.29 & $.36^{*}$ & -.20 & .22 \\
\hline 2. Sleep function & $.63 * * *$ & $-.45 * *$ & -.20 & -.29 & -.22 & $.33^{*}$ & -.09 & .04 \\
\hline 3. Ideal sleep & $.39 *$ & -.26 & -.01 & -.30 & $-.33^{*}$ & .30 & .17 & -.04 \\
\hline 4. Sleep last night & -.12 & -.17 & $-.46 * *$ & -.14 & .00 & .12 & $-.37 *$ & .28 \\
\hline 5. Sleep important & .18 & -.34 & .04 & -.23 & -.28 & .29 & -.02 & -.16 \\
\hline 6. Rate SD & $.31 *$ & $.39 *$ & $.32 *$ & .24 & .19 & $-.32 *$ & $.36 *$ & $-.32 *$ \\
\hline 7. ICD MNF & .16 & .02 & $.93 * * *$ & -.03 & -.12 & -.21 & .11 & .02 \\
\hline 8. ICD MWF & -.05 & $.78 * * *$ & -.25 & .63 & $.71 * * *$ & $-.48 * *$ & -.04 & .14 \\
\hline 9. ICD MST & $-.37 *$ & $-.43 * *$ & $-.43 * *$ & -.23 & -.14 & $.47 * *$ & -.07 & .02 \\
\hline 10. Maternal SD & - & -.09 & .18 & -.10 & -.12 & -.07 & -.04 & .05 \\
\hline 11. ICD MWT & -.09 & - & -.05 & $.66^{* * *}$ & $.55 * * *$ & $-.60 * * *$ & .11 & -.10 \\
\hline 12. ICD MNT & .18 & -.05 & - & -.05 & -.16 & -.22 & .10 & .01 \\
\hline 13. ICD BWT & -.10 & $.66^{* * *}$ & -.05 & - & $.85 * * *$ & $-.59 * *$ & .15 & .15 \\
\hline 14. ICD BWF & -.12 & $.55 * *$ & -.16 & $.85 * * *$ & - & $-.47 * * *$ & -.04 & .26 \\
\hline 15. ICD BST & -.07 & $-.60 * *$ & -.22 & $-.59 * * *$ & $-.47 * *$ & - & -.09 & .05 \\
\hline 16. BDI-II & -.04 & .12 & .10 & .15 & -.04 & -.09 & - & $-.47 * *$ \\
\hline 17. LOT-R & .04 & -.10 & .01 & .15 & .26 & .05 & $-.47 * *$ & - \\
\hline
\end{tabular}

Note. $\mathrm{SD}=$ sleep deprivation; ICD = Infant Care Diary; MNF = mom nap frequency; $\mathrm{MWF}=$ mom wake frequency; $\mathrm{MST}=$ mom sleep total; $\mathrm{MWT}=$ mom wake total; $\mathrm{MNT}=$ mom nap total; BWT = baby wake total during maternal sleep; BWF = baby wake frequency during 
maternal sleep; BST = baby sleep total; BDI-II = Beck Depression Inventory-II total score; LOT$\mathrm{R}=$ Life Orientation Test Revised total score.

${ }^{*} p<.05 .{ }^{* *} p<.01 .{ }^{* * *} p<.001$. 
Table 15

Intercorrelations Between Maternal and Principal Investigator (PI) Behavior Rating Scale Scores

\begin{tabular}{|c|c|c|c|c|}
\hline Measure & $\begin{array}{c}\text { Mom emotion } \\
\text { regulation }\end{array}$ & Mom motor & Mom orientation & Mom total \\
\hline $\begin{array}{l}\text { PI emotion } \\
\text { regulation }\end{array}$ & $.55 * * *$ & & & \\
\hline PI motor & & .26 & & \\
\hline PI orientation & & & $.53 * * *$ & \\
\hline PI total & & & & $.53^{* *}$ \\
\hline
\end{tabular}


Table 16

Means and Standard Deviations for Maternal Perceptions of Infant Manageability as a Function of Maternal Mood and Optimism

\begin{tabular}{llccc}
\hline & & & & \\
\multicolumn{1}{c}{ Optimism } & Depression & $M$ & $S D$ & $n$ \\
\hline High & High & 2.00 & 1.41 & 8 \\
& Low & 1.53 & .52 & 15 \\
Low & High & 2.13 & 1.41 & 3 \\
& Low & 1.67 & 1.15 & 3 \\
\hline
\end{tabular}


Table 17

Means and Standard Deviations for Maternal Expectations as a Function of Maternal Mood and Optimism

\begin{tabular}{llccc}
\multicolumn{1}{c}{ Optimism } & Depression & $M$ & $S D$ & $n$ \\
\hline High & High & .60 & .10 & 8 \\
& Low & .62 & .11 & 15 \\
Low & High & .64 & $8.55 \mathrm{E}-02$ & 3 \\
& Low & .65 & .20 & 35 \\
\hline
\end{tabular}


Table 18

Maternal Sleep Behavior Differences Between Mothers of First-Borns and Mothers of LaterBorns

$\underline{\text { First-borns }} \quad \underline{\text { Later-borns }}$

\begin{tabular}{lccccccc}
\multicolumn{1}{c}{ Measure } & $M$ & $S D$ & $n$ & $M$ & $S D$ & $n$ & $t(38)$ \\
\hline Wake total & .75 & .57 & 17 & .77 & .57 & 23 & -.12 \\
Wake frequency & 1.50 & 1.00 & & 1.58 & 1.09 & -.22 \\
Sleep total & 7.27 & .95 & & 6.54 & 1.07 & $2.23^{*}$ \\
Sleep deprivation & -9.03 E-02 & 1.19 & & .12 & 1.08 & -.57 \\
\hline
\end{tabular}

$* p<.05$ 
Figure 1. A model of the relations between infant night awakening and maternal and infant characteristics. 


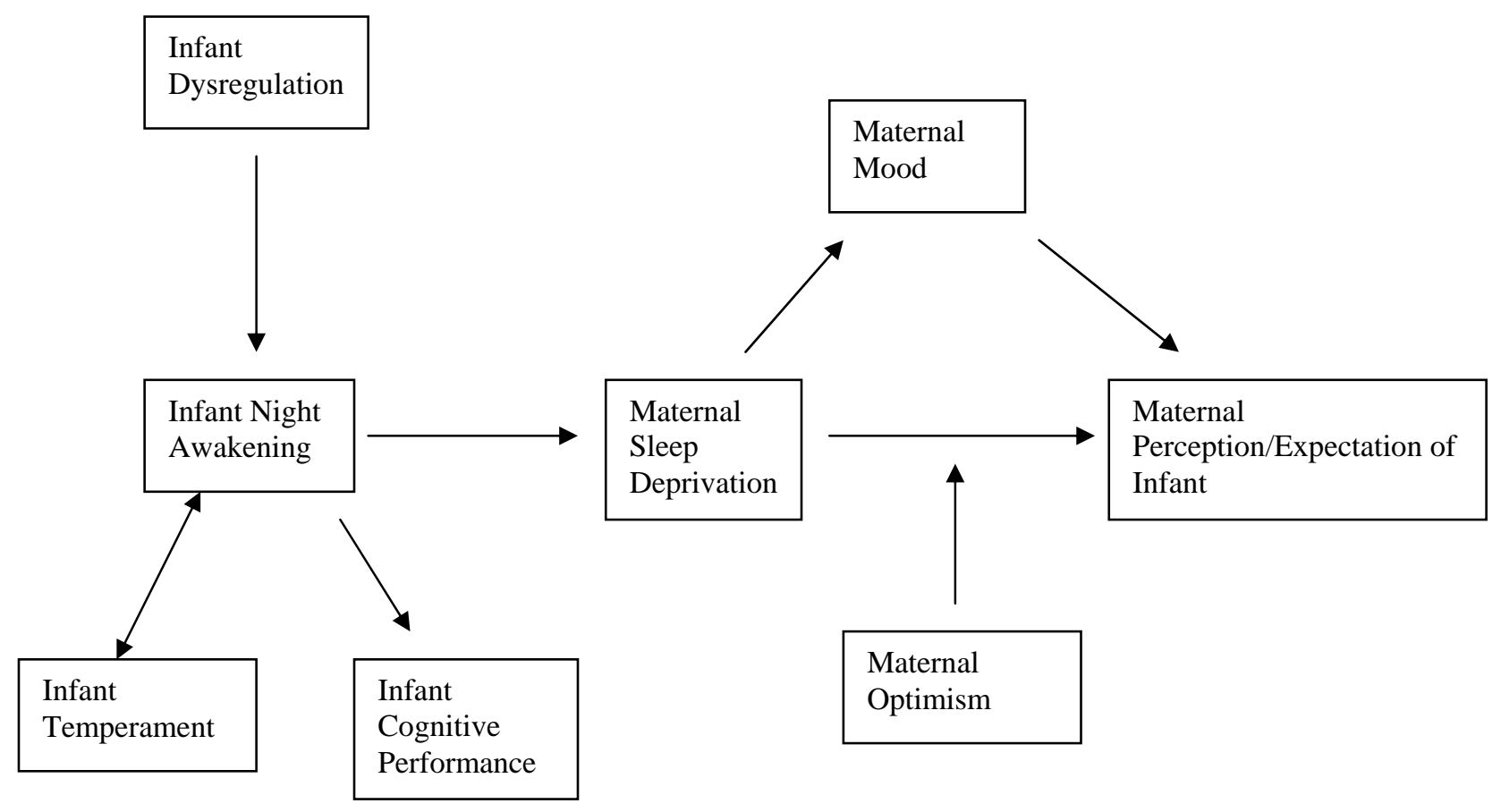


Appendix A 
Subject \#:

Date:

Time of Appointment:

\section{Information about you}

1. Current employment status:

\section{Background Questionnaire}

Full time: \# of hours per week:

Part time: \# of hours per week:

Volunteer: \# of hours per week:

Homemaker

2. Please list your occupation/job title/:

3. Please indicate your current level of education:

Without high school diploma

_ High school graduate, no college

_ Some college education

_ 4-year college degree

Some graduate school

Graduate degree

(specify degree:

4. If you are not currently working, do you plan on returning to work? Yes/No (please circle) If so, when? When my baby is months/years old (circle one).

5. Rate your general mood today:

$\begin{array}{ccccc}1 & 2 & 3 & 4 & 5 \\ \text { very negative } & & & & \text { very positive }\end{array}$

6. How many hours of sleep per night do you get on average?

7. Please indicate the number of hours of sleep you typically get on weeknights and weekends

8. What time do you usually get up in the morning? weeknight and weekend

9. What time do you usually go to bed at night? weeknight and weekend

10. How many nights per week is your sleep interrupted on average? (out of 7) 
11. How many nights per week is your sleep interrupted by:

__ your baby waking you another child waking you your husband/partner waking you not being able to sleep other

Please describe:
Typical length of interruption: $\mathrm{sec} / \mathrm{min} / \mathrm{hrs}$ (circle one) $\mathrm{sec} / \mathrm{min} / \mathrm{hrs}$ (circle one) $\mathrm{sec} / \mathrm{min} / \mathrm{hrs}$ (circle one) $\mathrm{sec} / \mathrm{min} / \mathrm{hrs}$ (circle one) $\mathrm{sec} / \mathrm{min} / \mathrm{hrs}$ (circle one)

12. How much sleep do you need to function well?

13. How much sleep per night would be ideal for you?

14. How much sleep did you receive last night?

15. How many times was your sleep interrupted last night by:

Typical length of interruption:

___ your baby waking you another child waking you your husband/partner waking you not being able to sleep other

Please describe: $\mathrm{sec} / \mathrm{min} / \mathrm{hrs}$ (circle one) $\mathrm{sec} / \mathrm{min} / \mathrm{hrs}$ (circle one) $\mathrm{sec} / \mathrm{min} / \mathrm{hrs}$ (circle one) $\mathrm{sec} / \mathrm{min} / \mathrm{hrs}$ (circle one) $\mathrm{sec} / \mathrm{min} / \mathrm{hrs}$ (circle one)

16. How important is it to you that you get enough sleep?

1 not important
2
3
5

very important

17. Do you regularly take naps? Yes/No (please circle) If so, please indicate how many times per week you take a nap on average.

18. Do you ever fall asleep while feeding your baby? Yes/No (please circle) If so, please indicate how many times per week you fall asleep while feeding your baby and for how long minutes.

19. Rate your current level of sleep deprivation:

$\begin{array}{ccccc}1 & 2 & 3 & 4 & 5 \\ \text { not sleep } & & & \begin{array}{c}\text { very sleep } \\ \text { deprived }\end{array}\end{array}$


20. To what extent do you think your current everyday functioning is negatively affected by sleep deprivation?

1

not at all
3

4

a great deal

21. To what extent do you think that sleep deprivation is interfering with your ability to interact with your baby?

$\begin{array}{ccccc}1 & 2 & 3 & 4 & 5 \\ \text { not at all } & & & & \text { a great deal }\end{array}$

\section{$\underline{\text { Information about your baby }}$}

22. When was your child born (month/day/year):

23. Please indicate your child's gender (circle one): Male Female

24. What time did your baby last awaken from a nap (indicate the time)?

25. How long was your baby's nap (indicate the length)?

26. If you baby has not had a nap today, what time did your baby awaken this morning?

27. When was your baby last fed (please indicate the time)?

28. Does your baby attend day care? Yes/No

29. If your baby does attend day care, how many hours per week does he/she attend?

30. Please indicate the approximate time that you put your baby to bed each night:

31. When you put your baby to bed is he or she typically awake or asleep? (circle one)

32. Where does your baby typically sleep at night?

_ Bassinet or crib in parents' room

_ Bassinet or crib in own room

_ Bassinet or crib in sibling room

_ In bed with ___ (please indicate)

_ Other or combination (please describe)

33. Does your baby co-sleep with you (i.e. does your infant sleep in bed with you)? Yes/No

34. If your infant does co-sleep with you, how many times per week is he or she in bed with you? and on average how many hours does he/she spend sleeping with you? 
35. Does your infant sleep with a sleep aid (i.e. pacifier, blanket, stuffed animal)? Yes/No If so, please indicate what:

36. Please check which scenario applies to your infant and indicate at what age the following occurred:

My infant has continuously slept through the night since he or she was months old and rarely/sometimes needs my help to fall back asleep after he or she awakens during the night.

My infant has frequently awakened during the night since he or she was months old and usually/always needs my help to fall back asleep after he or she awakens during the night.

My infant started sleeping through the night when he or she was months old but started awakening during the night again when he or she was months and usually/always needs my help to fall back asleep after he/she awakens during the night. Other (please describe):

37. Please indicate the number of times your baby typically awakens each night:

38. Who typically cares for your baby when he or she awakens during the night?

39. If you share nighttime care, what percent of the time do you care for your baby when he or she awakens during the night?

40. Please indicate what you do to get your baby back to sleep during the night:

41. Does your baby show distress when separated from you? Yes/No (please circle)

42. If your baby shows distress when separated from you at what age did that behavior begin? months

43. If your baby shows distress when separated from you, rate the intensity of your baby's distress at separation:

1

low
2

3
45

high

44. Is your infant currently teething? Yes/No

45. If your infant is currently teething, please rate the amount of fussiness/crying your infant experiences as a result:

1

little fussing/crying
2

3
4 much fussing/crying 


\section{Information about your household:}

46. Please list the relation to your baby of all the people living in your home (i.e. father, older siblings, relatives):

47. If you are living with a husband/partner, please indicate on average how much sleep your husband/partner gets at night:

48. If you are living with a husband/partner, please indicate how many times your husband/partner awakens during the night on average:

49. If you are living with a husband/partner, what is your husband/partner's job title/occupation? (Please indicate if unemployed)

50. Please indicate your husband/partner's current education level:

_ Without high school diploma

_ High school graduate without college education

_ Some college education

_ 4-year college degree

_ Some graduate school

__Graduate degree (specify degree:

51. Please indicate your race/ethnicity:

Non-Hispanic White

Hispanic

African American

Native American

Asian/Pacific Islander

Other (please indicate):

52. Please indicate your husband/partner's race/ethnicity:

Non-Hispanic White

Hispanic

African American

Native American

Asian/Pacific Islander

Other (please indicate):

53. Please report your approximate yearly household income:

54. What is your current age?

55. Do you breast or bottle feed your baby? (circle one) 
Appendix B 


\section{Participant No.}

Date

\section{INFANT CARE DIARY \\ (postnatal version)}

The Infant Care Diary is designed to assess your own and your baby's typical sleep patterns and your infant's eating and fussing/crying patterns. Please complete this diary for one week, beginning at noon on a Monday. Each page (front and back) can be used for one 24-hour period. The diary is marked off in 15-minute intervals. Mark an X in any interval during which you slept most of the time. For naps during the day, write NAP instead of X. Use $\mathrm{X}$ to designate night-time sleep. Whenever you awaken from having been asleep (for more than a few minutes), please write the reason you awakened. Examples of reasons include baby signaling (fuss/cry/vocalize), alarm going off, spontaneous waking, need to go to the bathroom, bed partner snoring, physical discomfort, noise, etc.

Also mark with Xs the times your baby sleeps, fusses or cries, and eats. Mark any 15-minute interval during which your baby fusses or cries or eats at all. For sleeping, mark an interval if the infant slept for most of the interval. If you are away from your baby during the week, please ask the person caring for your baby to record these behaviors. If they are unwilling or unable to do this, please indicate on the chart the times when you don't know what your baby did.

If you have any questions about how to complete this diary, please call Cheryl (293-2001, extension, 31625) or Dr. Karraker (293-2001, extension 31625).

Please remember to complete the packet of questionnaires during the week you are completing this diary. Thanks for your help! 
Monday noon to midnight

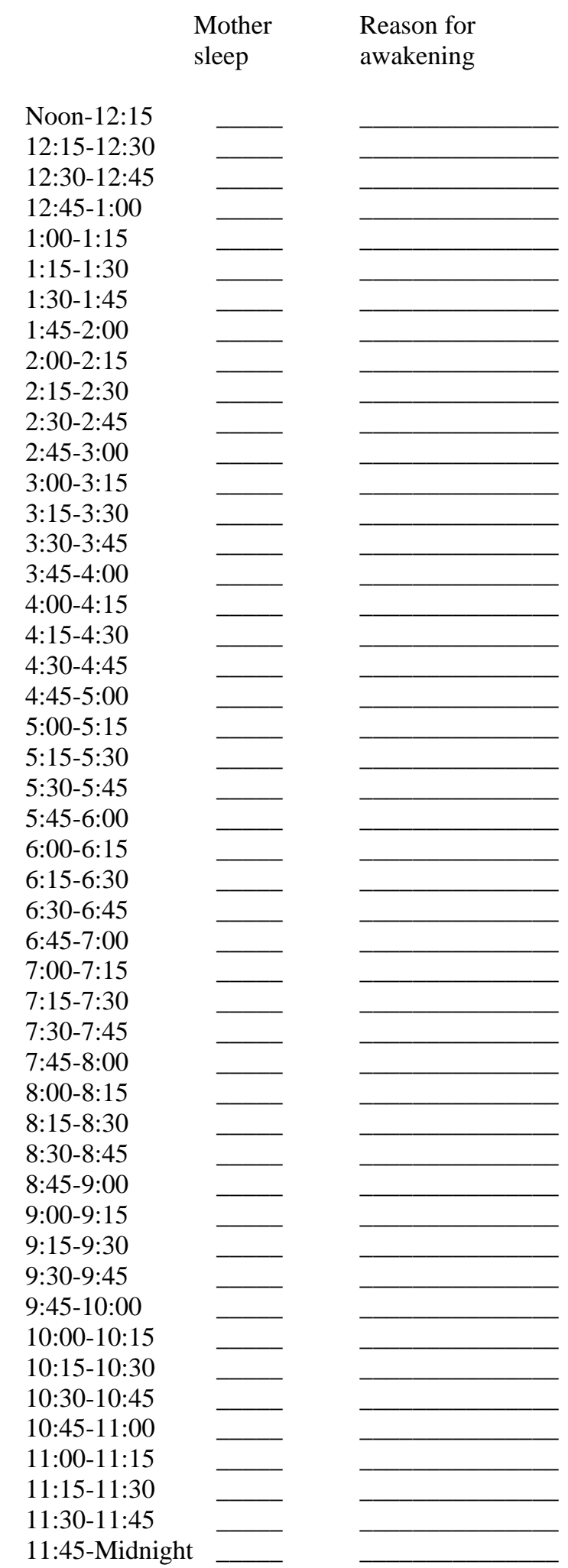

Date this page started

$\begin{array}{llll}\text { Baby } & \text { Baby } & \text { Baby } & \text { Not } \\ \text { sleep } & \text { fuss/cry } & \text { eat } & \text { known }\end{array}$

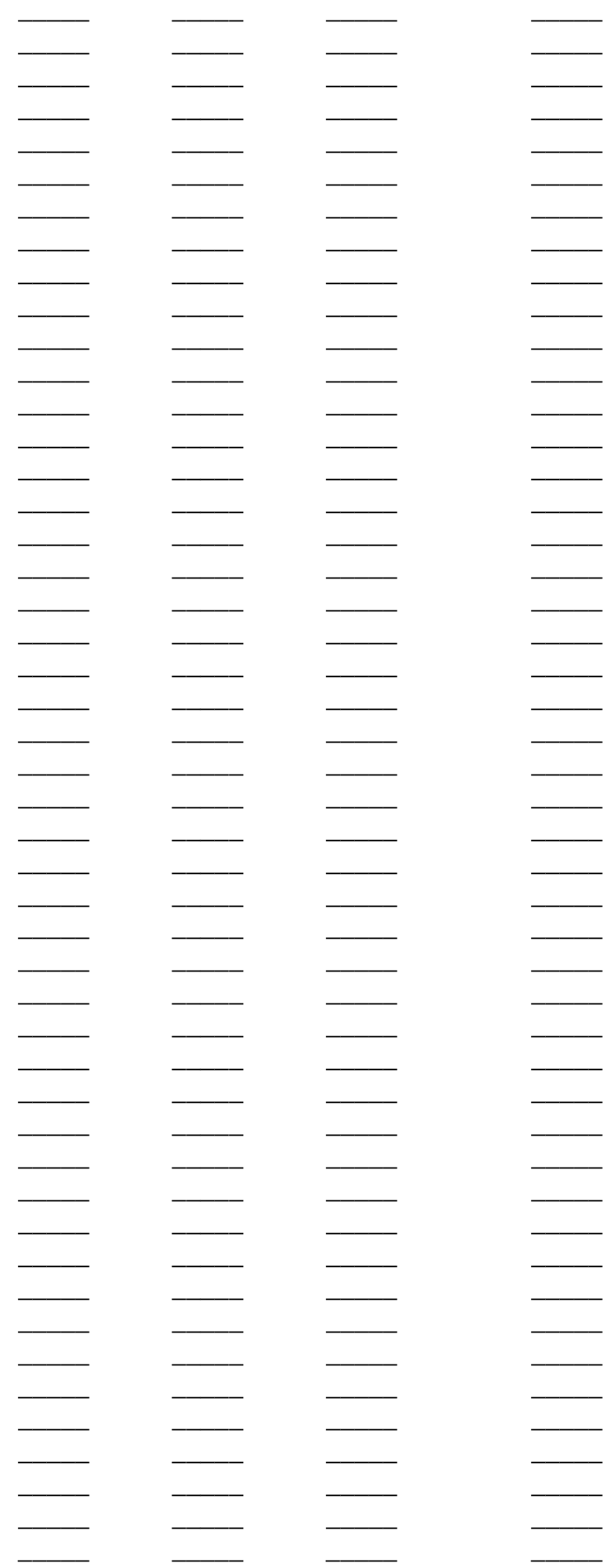


Appendix C 
Subject Number:

Date:

\section{Bayley Expectations Questionnaire - 9 months}

We will be administering the items described and illustrated below from the Bayley Scales of Infant Development to your infant. We are interested in your expectations concerning your infant's ability to perform these items. For each item, please predict whether or not your infant will be able to do the action described. If you do not understand an item from the description, ask the test administrator for further explanation or a demonstration of the item.

1. Rings bell purposefully. The examiner will ring a bell and then set it within your infant's reach.

Will your infant ring the bell?

YES

NO

2. Pulls string adaptively to secure ring, suspends ring by string. The examiner will dangle a plastic ring (about 4" in diameter) by an attached string and then place it on the table.

Will your infant pull the string and get the ring? $\quad$ YES NO Will your infant pick up and dangle the ring by the string? YES NO

3. Retains two of three cubes for 3 seconds, attempts to secure three cubes. The examiner will place 3 small blocks on the table. If your infant does not pick up two of the blocks the examiner will place them in your infants' hands.

Will your infant hold two blocks for at least 3 seconds?

YES

NO

Will your infant try to pick up a third block while holding the other two?

YES

NO

4. Lifts cup by handle. The examiner will hide a small toy under a plastic tea cup.

Will your infant pick up the cup by the handle?

YES

NO

5. Puts one block in cup. The examiner will place a block inside a cup and then give your infant the cup and a number of blocks.

Will your infant put one block in or over the cup, even if he/she does not release it?

YES

NO

Will your infant put at least three blocks in or over the cup, even YES

NO if he/she does not release it? 
6. Finds one object. The examiner will hide an object underneath one or two inverted cups, alternating between each cup on three occasions. The examiner will ask your infant to find the object each time.

Will your infant find the object at least two times?

YES

NO

7. Looks for contents of a box. The examiner will place two small beads in a plastic box, pour them out, then put them back in, then remove them under the table (so your infant cannot see them being removed), and hand the box to your infant.

Will your infant look inside the box for the beads?

YES

NO

8. Removes lid from a box. The examiner will place a small toy inside a small plastic box and place the lid on the box. Then, the examiner will take the lid off, remove the toy, show the toy to your infant, put the toy back in the box, replace the lid, and place the box in front of your infant.

Will your infant be able to remove the lid in at least two out of three tries?

YES

NO

9. Pushes car. The examiner will push a small toy car across the table and give the car to your infant.

Will your infant then push the car?

YES

NO

10. Fingers holes in a pegboard. The examiner will place a pegboard (without pegs) in front of your infant and show your infant how to poke a finger in the holes.

Will your infant then poke his/her finger into at least one hole? YES

NO

11. Looks at pictures in book, turns pages of book. The examiner will place an open picture book in front of your infant.

Will your infant look at the pictures?

YES

NO

Will your infant turn the pages of the book?

YES

NO

12. Pats toy in imitation. The examiner will place a toy in front of your infant and pat it to make a whistling sound and then encourage your infant to do the same.

Will your infant imitate the patting motion?

YES

NO

13. Imitates vocalization. Either your or the examiner will make some sounds that your infant typically makes (like baba or dada).

Will your infant imitate the sounds?

YES

NO 
14. Cooperates in a game. The examiner will play peek-a-boo with your infant. Will your infant actively participate in the game? YES

15. Listens selectively to two familiar words.

During the test, will your infant respond to the sound of at least two familiar words (i.e. by changing facial expressions, vocalizing, or imitating the words) YES

NO

16. Responds to spoken request. The examiner will ask you to ask your infant to perform some action, such as "Show me your nose" or "Where is the light?"

Will your infant respond appropriately to your request?

YES

$\mathrm{NO}$

17. Removes sugar pellet from bottle. The examiner will place a sugar pellet in a bottle and instruct your infant to remove it.

Will your infant be able to purposefully remove the pellet

from the bottle?

YES

$\mathrm{NO}$

18. Uses gestures to make wants known.

During the test, will your infant use a gesture (like reaching, pointing, or pushing something) to communicate with you or the examiner? YES

19. Repeats vowel-consonant combination.

During the test, will your infant spontaneously say at least one vowel-consonant combination (like da-da, ba-ba, ma-ma etc...)?

YES

NO

20. Jabbers expressively.

During the test, will your infant jabber expressively at least once? YES

NO

21. Vocalizes four different vowel-consonant combinations.

During the test, will your infant vocalize at least four different vowel-consonant combinations (like da, ma, ba, goo, um, uh, an etc...)?

YES

NO

22. Motor skills. Will your infant be able to demonstrate the following skills?

Rotates trunk (turns to the side to reach for a toy)

while sitting alone.

YES

NO 
Pick up a small pellet (made of sugar) by using his/her thumb and the rest of his/her hand.

YES

NO

Uses pads of fingertips to grasp pellet.

YES

NO

Grasps a rod using his/her thumb and the rest of his/her hand.

Grasps a pencil at either the farthest or nearest end

(not the middle).

Moves from a sitting to a creeping position

Raises him/herself to a standing position from a lying position using any available support.

Attempts to walk while standing with his/her hands held.

Walks sideways while holding on to furniture.

Stands up from a position lying on his/her back without using any support.

Walks with help (one hand on an adult or furniture).

Stands alone without help for at least 2 seconds.

Walks alone for at least 3 steps without help.

Sits down (in a controlled manner) from standing (with or without support).
YES

YES

NO

YES

NO

YES

NO

YES

NO

YES

NO

YES

NO

YES

NO

YES

NO

YES

NO 
Appendix D 


\begin{tabular}{|c|c|c|}
\hline Infant performance & Mother's expectation for & infant performance \\
\hline & PASS & FAIL \\
\hline PASS & A & B \\
\hline FAIL & C & D \\
\hline
\end{tabular}

$\mathrm{A}+\mathrm{B} /(\mathrm{A}+\mathrm{B}+\mathrm{C}+\mathrm{D})=$ proportion of infant passes

$A+C /(A+B+C+D)=$ proportion of expected passes

$\mathrm{A}+\mathrm{D} /(\mathrm{A}+\mathrm{B}+\mathrm{C}+\mathrm{D})=$ proportion of correct expectations

$\mathrm{B} / \mathrm{A}+\mathrm{B}=$ proportion of underestimations

$\mathrm{C} / \mathrm{C}+\mathrm{D}=$ proportion of overestimations 